Meta

Journal des traducteurs

Translators' Journal

\title{
Le traitement de la modalité épistémique dans les traductions françaises de On the Origin of Species de Charles Darwin
}

\section{Eve-Marie Gendron-Pontbriand}

Volume 61, numéro hors-série, 2016

Sciences en traduction

Sciences in Translation

URI : https://id.erudit.org/iderudit/1038687ar

DOI : https://doi.org/10.7202/1038687ar

Aller au sommaire du numéro

Éditeur(s)

Les Presses de l’Université de Montréal

ISSN

0026-0452 (imprimé)

1492-1421 (numérique)

Découvrir la revue

Citer cet article

Gendron-Pontbriand, E.-M. (2016). Le traitement de la modalité épistémique dans les traductions françaises de On the Origin of Species de Charles Darwin. Meta, 61, 87-112. https://doi.org/10.7202/1038687ar
Résumé de l'article

Éminent naturaliste du XIX ${ }^{\mathrm{e}}$ siècle, Charles Darwin publie en 1859 ce qui s'avérera être un des textes les plus fondamentaux des sciences de la vie : On the origin of species by means of natural selection, or the preservation of favoured races in the struggle for life (ou OS). Cet ouvrage pose les assises conceptuelles de sa théorie de l'évolution (TE). Or, malgré la grande portée de l'oeuvre, ses traductions en français restent largement inexplorées, en traductologie comme en histoire des sciences. Les quelques travaux antérieurs sur le sujet se sont concentrés sur les traductions de Clémence Royer, à qui l'on reproche d'avoir radicalisé la TE darwinienne en transformant la prudence de Darwin en certitude. Présentement, c'est pourtant la traduction d'Edmond Barbier (1876), et non celles de Royer, qui fait autorité ; trois autres traducteurs, deux contemporains de Darwin et un moderne, se sont également attaqués à l'OS. Nous nous proposons donc de comparer ces différentes traductions, avec leur texte original, mais également entre elles, sur la base de marqueurs de modalité épistémique précis qui atténuent le degré de certitude d’un énoncé et qui sont très caractéristiques du style particulièrement précautionneux de Darwin. Nous voulons ainsi déterminer comment ces autres traducteurs ont rendu la modalité du texte anglais et montrons que la version la plus moderne est la plus proche de l'original. Enfin, de manière inattendue, la traduction de Royer, depuis longtemps décriée par la critique, se rapproche plus des traductions de Moulinié et de Barbier qu'on ne l'anticipait.
Ce document est protégé par la loi sur le droit d'auteur. L’utilisation des services d’Érudit (y compris la reproduction) est assujettie à sa politique d'utilisation que vous pouvez consulter en ligne.

https://apropos.erudit.org/fr/usagers/politique-dutilisation/ 


\title{
Le traitement de la modalité épistémique dans les traductions françaises de On the Origin of Species de Charles Darwin
}

\author{
EVE-MARIE GENDRON-PONTBRIAND \\ Université de Montréal, Montréal, Canada \\ eve-marie.gendron-pontbriand@umontreal.ca
}

\begin{abstract}
RÉSUMÉ
Éminent naturaliste du XIXe siècle, Charles Darwin publie en 1859 ce qui s'avérera être un des textes les plus fondamentaux des sciences de la vie: On the origin of species by means of natural selection, or the preservation of favoured races in the struggle for life (ou OS). Cet ouvrage pose les assises conceptuelles de sa théorie de l'évolution (TE). Or, malgré la grande portée de l'œuvre, ses traductions en français restent largement inexplorées, en traductologie comme en histoire des sciences. Les quelques travaux antérieurs sur le sujet se sont concentrés sur les traductions de Clémence Royer, à qui l'on reproche d'avoir radicalisé la TE darwinienne en transformant la prudence de Darwin en certitude. Présentement, c'est pourtant la traduction d'Edmond Barbier (1876), et non celles de Royer, qui fait autorité; trois autres traducteurs, deux contemporains de Darwin et un moderne, se sont également attaqués à l'OS. Nous nous proposons donc de comparer ces différentes traductions, avec leur texte original, mais également entre elles, sur la base de marqueurs de modalité épistémique précis qui atténuent le degré de certitude d'un énoncé et qui sont très caractéristiques du style particulièrement précautionneux de Darwin. Nous voulons ainsi déterminer comment ces autres traducteurs ont rendu la modalité du texte anglais et montrons que la version la plus moderne est la plus proche de l'original. Enfin, de manière inattendue, la traduction de Royer, depuis longtemps décriée par la critique, se rapproche plus des traductions de Moulinié et de Barbier qu'on ne l'anticipait.
\end{abstract}

\begin{abstract}
In 1859, prominent British naturalist Charles Darwin publishes On the origin of species by means of natural selection, or the preservation offavoured races in the struggle for life (or OS). In this book, Darwin introduces his theory of evolution (TE), which has been shrouded in controversy since its inception. Yet, despite the book's profound impact on the world at large, its French translations have rarely been studied. The few existing studies have focussed on translations by Clémence Royer, a controversial translator infamous for having coopted Darwin's writings to serve a more radical agenda. But the fact remains that the canonical French translation of the OS is the one by Edmond Barbier, not Royer; three translators, two contemporary to Darwin and one modern, have also translated the book. Therefore, we aim to compare and contrast these various French translations with their original text and amongst each other on the basis of hedges, epistemic modality markers that attenuate the degree of certainty associated with a statement. We wish to determine how each translator has expressed epistemic modality in his/her text and show that the modern translation is closest to the original. Unexpectedly, Royer's translation, long decried by critics, is closer to Moulinié's and Barbier's translations than previously anticipated.
\end{abstract}

\section{MOTS CLÉS/KEYWORDS}

rhétorique scientifique, modalité épistémique, modalité épistémique atténuatrice, annotation sémantique, traduction scientifique

scientific rhetoric, epistemic modality, hedging, semantic annotation, scientific translation 
Ignorance more frequently begets confidence than does knowledge: it is those who know little, not those who know much, who so positively assert that this or that problem will never be solved by science.

$(\text { Darwin } 1871: 3)^{1}$

\section{Introduction}

Les textes scientifiques sont réputés être de simples vecteurs d'un contenu référentiel, impersonnels et objectifs (Olohan et Salama-Carr 2011: 179). Hyland (1999: iii) affirme au contraire que l'écriture scientifique est hautement rhétorique. L'utilisation de marqueurs de modalité épistémique atténuateurs (MMEA; hedges en anglais) est l'une des stratégies rhétoriques les plus courantes, notamment dans les textes scientifiques, car ils permettent aux auteurs d'exprimer la possibilité, l'hypothèse, la prudence voire le doute, plutôt que la certitude. Crismore et Farsnworth (1989) ont montré que ces marqueurs d'atténuation se retrouvent en grande quantité dans une œuvre scientifique qui a fait époque: On the origin of species by means of natural selection, or the preservation of favoured races in the struggle for life $e^{2}$ (dorénavant OS), ouvrage phare de l'éminent naturaliste britannique Charles Robert Darwin (1809-1882), dont la première édition est parue en 1859. Ces marqueurs constituent donc un élément caractéristique du texte darwinien. Leur importance tient au fait qu'ils témoignent de la volonté de Darwin de se montrer prudent, circonspect, modeste, bref, diplomate, en raison du caractère révolutionnaire des idées qu'il avance dans l'OS (Crismore et Farsnworth 1989: 101). De plus, bien que Brisset (2002) ait montré que le traitement de ces marqueurs dans la traduction française de l'OS par Clémence Royer (Darwin 1861/1862) est problématique, on sait très peu de choses des autres traductions des différentes éditions de l'OS, car elles n'ont pas été étudiées. Afin de combler ce vide, nous avons entrepris une étude quantitative comparative des marqueurs de modalité épistémique dans quatre traductions françaises de l'OS.

\section{Contexte et problématique}

C'est dans l'OS que Darwin pose les assises conceptuelles de sa théorie de l'évolution (TE). Pendant les 150 ans qui ont suivi la parution de l'OS, la TE a révolutionné notre compréhension du vivant et de ses origines d'une manière qui dépasse largement les frontières de la science. En 1973, le célèbre biologiste Theodosius Dobzhansky ${ }^{4}$ publie un article au titre évocateur: Nothing in Biology Makes Sense Except in the Light of Evolution ${ }^{5}$. Maintes fois repris et cité, ce titre illustre bien la place privilégiée qu'occupe aujourd'hui encore la TE au sein de la pensée scientifique dans sa version moderne dite «synthétique» ou "néodarwinienne». En effet, elle constitue un des principaux fondements théoriques de l'ensemble des sciences de la vie: biologie, génétique, écologie, médecine, paléontologie, anthropologie, pour n'en citer que quelques-unes. Dans la sphère publique, cette théorie fait toutefois encore l'objet de nombreux débats; certains la nient (ceux qui prônent notamment le créationnisme et le dessein intelligent) alors que d'autres la poussent à l'extrême (ceux qui font entre autres la promotion de l'eugénisme et du darwinisme social). Ainsi, le sujet est toujours d'actualité et suscite encore beaucoup d'intérêt. 
L'OS est une œuvre d'une très grande envergure, tant en volume que dans le temps, et de surcroit très complexe. En effet, on compte six éditions anglaises originales ${ }^{6}$, toutes parues chez l'éditeur John Murray (Londres). Près de vingt ans séparent la première et la dernière. Durant ce temps, voulant répondre à ses détracteurs, Darwin a modifié le texte de façon substantielle, en y apportant de nombreuses additions, suppressions et corrections. L'OS a également été très largement traduite (et souvent retraduite), et ce, dans au moins une trentaine de langues, y compris le français ${ }^{7}$.

Pourtant, les traductions de l'OS, notamment les traductions françaises qui nous intéressent ici, demeurent pratiquement inexplorées (voir Vandaele et GendronPontbriand 2015). Les quelques études qui se sont penchées sur les traductions françaises de l'OS portent exclusivement sur celles, controversées, de Clémence Royer. Sa traduction de la $3^{\mathrm{e}}$ édition anglaise (voir note 3), publiée en 1862, est la première traduction française de l'œuvre. Dans son Introduction du darwinisme en France au $X I X^{e}$ siècle (1974), Conry critique fortement Royer et sa traduction, l'accusant d'avoir volontairement détourné le texte de Darwin pour servir ses convictions eugénistes personnelles. Pour sa part, Miles $(1988 ; 1989)$ tente de défendre Royer. Ayant analysé sa traduction, elle identifie, de fait, de nombreuses «divergences» entre la traduction de Royer et l'original anglais, notamment des changements stylistiques et des glissements qu'elle qualifie d'ordre "philosophique», mais elle conclut que la transformation résultante du texte darwinien - d'un style plus prudent vers un style plus affirmatif - était justifiée pour rendre le texte acceptable pour un auditoire français.

Cherchant à trouver un juste milieu entre les positions de Conry et Miles sur la qualité de la traduction de Royer, Brisset (2002) se place en médiatrice entre les deux auteures. Elle décrit la vie et l'œuvre de cette intellectuelle, autodidacte et polymathe, qui défendait ardemment les droits des femmes, mais également une certaine forme d'eugénisme et de darwinisme social. Moins dure à l'endroit de Royer que Conry, Brisset n'en demeure pas moins critique à l'issue de sa propre analyse de la traduction de Royer. Elle conclut que l'expression du doute y est modifiée - ce qui est présenté comme probable dans l'original anglais tend à devenir certain dans la traduction d'une manière qui dépasse l'adaptation aux normes françaises. Ce faisant, Royer ne rend pas justice à l'original darwinien.

En évoquant, respectivement, des «changements stylistiques» ou une modification de «l'expression du doute» dans la traduction de Royer par rapport à l'original anglais, Miles et Brisset se réfèrent en fait à l'emploi de la modalité épistémique atténuatrice (MEA, hedging en anglais; voir section 4), sans toutefois la nommer explicitement. Elles n'ont simplement pas eu recours à un cadre théorique linguistique pour la nommer et la décrire. Vandepitte, Vandenbussche, et al. (2011) ont pour leur part eu recours au concept de modalité épistémique dans leur étude de deux traductions néerlandaises de l'OS: celles de Tiberius Cornelius Winkler (Darwin 1859/1860) ${ }^{8}$ et de Ludo Hellemans (Darwin 1859/2000) ${ }^{9}$, toutes deux réalisées à partir de la première édition anglaise de l'OS (1859). Ces auteurs ont analysé la traduction de huit marqueurs modaux épistémiques (will, may, might, possibl ${ }^{\star}$, probabl ${ }^{\star}$ et certainly) et ont montré que les deux traductions présentaient des différences de traitement de ces marqueurs. En effet, le degré de certitude exprimé par l'original est conservé dans la traduction de Hellemans, alors qu'il est accentué dans celle de Winkler. L'intérêt de quantifier des marqueurs réside dans le fait que ceci permet de conclure avec plus de certitude quant aux changements apportés par la traduction. 
Par ailleurs, en ce qui concerne le français, il est aisé de comprendre pourquoi les traductions de Royer ont piqué l'intérêt des chercheurs: traitement des passages exprimant le doute, préface incendiaire, notes de traduction très détaillées, et enfin conflit entre Darwin et Royer (Darwin 1887: 110). Bien que cet intérêt soit justifié, le travail des autres traducteurs français qui se sont attaqués à l'OS n'a pourtant jamais été étudié. De fait, Conry et Miles ont abordé le sujet, respectivement, du point de vue de la philosophie des sciences et de l'histoire des sciences. S’intéressant à la traductrice plutôt qu'à sa traduction, l'article de Brisset avait quant à lui des visées sociobiographiques. Bien que nous ne remettions pas en question la finesse de l'analyse de ces trois auteures, ceci fait en sorte que les paramètres analysés n'ont pas été étudiés dans un cadre linguistique ni de manière quantitative.

Ainsi, la concentration des travaux antérieurs sur les traductions de Royer donne lieu à un portrait incomplet des traductions françaises de l'OS. Le présent article se propose de remédier à cette situation en se penchant sur le traitement des MMEA dans quatre traductions françaises de l'OS, celles de Royer (Darwin 1861/1862; à titre de point de comparaison; voir note 3), de Moulinié (Darwin 1869/1873) ${ }^{10}$, de Barbier (Darwin 1872/1876) ${ }^{11}$ et de Berra (Darwin 1876/2009) ${ }^{12}$, car nous nous interrogeons sur le traitement des MMEA par les autres traducteurs de l'OS. Nous commencerons par situer le concept de modalité épistémique au sein du domaine plus large de la modalité, avant de présenter la méthodologie, y compris la stratégie de délimitation du corpus, qui nous permettra de comparer les traductions avec le texte source, mais aussi entre elles. Nous terminerons par l'analyse statistique des données extraites du corpus et une discussion du traitement des MMEA dans chacune des traductions.

\section{La modalité: un aperçu}

La modalité fait l'objet de discussion depuis l'Antiquité: elle relève autant des domaines de la philosophie et de la logique formelle que de la psychologie et de la linguistique (Day 2008). Il s'agit d'un phénomène linguistique complexe et hétérogène, difficilement circonscrit. De façon générale, la modalité permet aux locuteurs d'une langue de faire référence à des situations hypothétiques ou non réelles (Ziegeler 2006). En d'autres mots, la modalité permet d'exprimer l'existence de «mondes possibles» (von Fintel et Gillies 2007). Portner (2009) souligne l'existence de nombreux types de modalité et présente deux façons de les classifier. La première classification se fait en fonction de la nature de la modalité (figure 1, axe vertical), alors que la deuxième s'intéresse plutôt à la portée de la modalité (figure 1, axe horizontal). Dans un premier temps, on distingue la modalité épistémique (epistemic modality) de la modalité déontique (deontic modality). La modalité épistémique porte sur les connaissances et les croyances: il s'agit du degré de certitude par rapport à la vérité d'un énoncé, exprimé par le locuteur lui-même. Au contraire, la modalité déontique relève du langage prescriptif et permet d'exprimer ce qui est bien et ce qui est mal, en fonction d'un ensemble de règles donné. Dans un deuxième temps, on distingue la modalité sous-phrastique (sub-sentential modality), la modalité phrastique (sentential modality) et la modalité discursive (discourse modality). La modalité sous-phrastique se situe au sein d'éléments plus restreints que la phrase, plus précisément, au sein de la proposition ${ }^{13}$, notamment la modalité inhérente au sens lexical de certains verbes (figure 1A), noms et adjectifs (figure 1D) ou aux modes verbaux. 
La modalité phrastique concerne un élément discret qui affecte le sens global de la proposition, entre autres les adverbes, les auxiliaires modaux (figure $1 \mathrm{~B}$ et $1 \mathrm{E}$ ), les temps verbaux ${ }^{14}$. Enfin, la modalité discursive met en jeu l'articulation du discours, et donc peut impliquer plus qu'une phrase, comme le type de phrase utilisée (figure $1 F$ : une phrase impérative), la modalité subordonnée (figure 1C: la modalité exprimée dans la première phrase ["peut-être»] affecte l'interprétation de la deuxième [Portner 2009: 8] $)^{15}$, etc.

FIGURE 1

Typologie croisée des types de modalité ${ }^{16}$

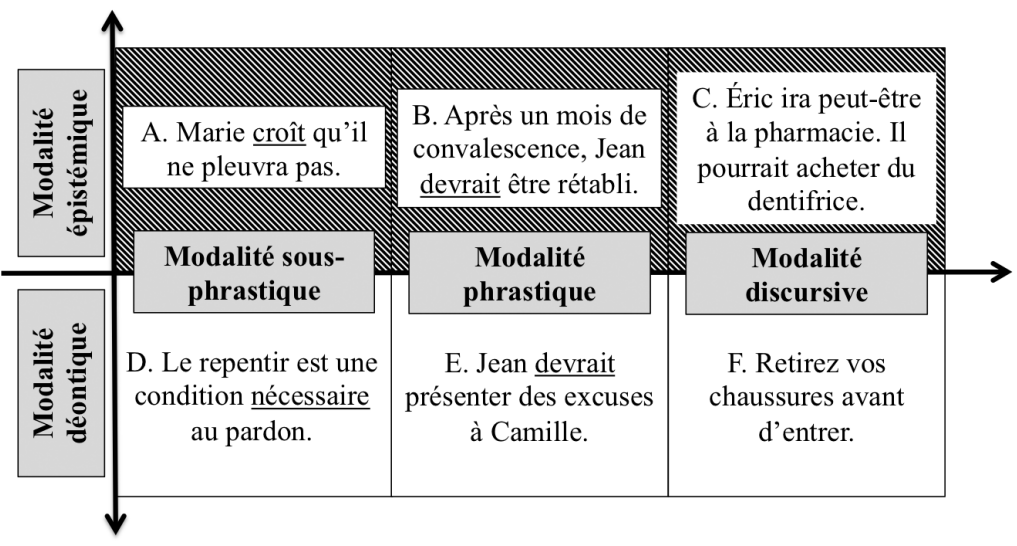

Nous nous sommes concentrée sur la modalité épistémique autant au plan sousphrastique que phrastique et discursif (voir les sections hachurées de la figure 1). La modalité déontique n'a pas été retenue, car on ne lui reconnaît pas de rôle rhétorique dans l'OS.

\section{Marqueurs de modalité épistémique: le modèle de Hyland}

La modalité épistémique est un phénomène complexe qui a fait l'objet de très nombreuses recherches, chacune l'abordant sous un angle particulier (Portner 2009; Day 2010). Elle permet au locuteur d'exprimer son attitude par rapport à la vérité de la situation qu'il énonce. Hyland (1999) s'attarde à la modalité épistémique atténuatrice et à ses marqueurs (MMEA). La MEA a une fonction particulière: "[Hedges are] used to indicate either a) a lack of complete commitment to the truth value of an accompanying proposition, or b) a desire not to express that commitment categorically» (Hyland 1999: 2). Ce qui est important ici est la marque d'atténuation. Ainsi, sont exclus des MMEA les marqueurs épistémiques exprimant le jugement de valeur d'un locuteur ("Les résultats étaient étonnamment clairs", «Il est terrifiant de penser que...») et ceux exprimant sa conviction de la vérité d'une proposition ("Les résultats montrent clairement que...», "Il est évident que...»).

Hyland établit également une typologie des MMEA, constituée de trois catégories. La MEA lexicale et la MEA syntaxique se réalisent respectivement sur le plan de l'unité lexicale et de la syntaxe. La MEA stratégique concerne le propos du locuteur. Le tableau 1 présente cette typologie, ainsi que différentes sous-catégories de 
MMEA et des exemples. Dans la présente étude, en raison de l'ampleur de l'analyse réclamée dans une œuvre aussi vaste que l'OS, nous nous sommes concentrée sur le repérage des MMEA syntaxiques et lexicaux. En effet, leur caractère d'unité quasi discrète autorise une méthode de repérage ciblant ces unités précisément. La MEA stratégique pourra faire l'objet d'une étude ultérieure, avec une méthode qui lui sera spécifique.

TABLEAU 1

Marqueurs relevant des trois catégories de MEA selon Hyland ${ }^{17}$

\begin{tabular}{|l|l|}
\hline MEA lexicale & Exemples \\
\hline Verbes lexicaux & indicate, assume, believe, imply, suggest \\
\hline Auxiliaires modaux & may, might, must, can, could, would \\
\hline Adverbes & likely, possibly, probably, generally \\
\hline Adjectifs & possible, approximate, relative \\
\hline Noms modaux & estimate, possibility \\
\hline MEA syntaxique & \multicolumn{2}{|l|}{ splenectomy was performed $[\ldots]$} \\
\hline Forme passive & could it not be that $[\ldots]$ ?... \\
\hline Phrase interrogative & it is not known whether... \\
\hline Phrase impersonnelle & \multicolumn{2}{|l|}{} \\
\hline MEA stratégique & under these conditions phosphorylation of PEPc by [...] \\
\hline Référence à des conditions limitantes & in the context of the proposed model $[\ldots]$ \\
\hline Référence à un consensus & there is no knowledge concerning $[\ldots]$ \\
\hline Aveu d'un manque de connaissance &
\end{tabular}

\section{Méthodologie}

L'étude des traductions françaises de l'OS sur le plan de la modalité épistémique a nécessité l'élaboration d'une méthodologie spécifique. Dans les prochaines soussections, nous décrirons la stratégie employée pour délimiter le corpus à l'étude, la méthode d'annotation du corpus, ainsi que les outils utilisés pour l'extraction et l'analyse des données.

\subsection{Délimitation $d u$ corpus}

Comme nous l'avons souligné plus haut, l'OS est une œuvre imposante et complexe. Nous décrivons, dans le tableau 2, les différentes éditions anglaises de l'OS publiées du vivant de Darwin. Chacune d'entre elles s'est vu attribuer un code d'identification que nous utiliserons dorénavant pour y faire référence. Par ailleurs, il existe à ce jour dix traductions françaises, qui comportent en moyenne 450 pages chacune (Vandaele et Gendron-Pontbriand 2015). Celles-ci, avec leur code d'identification, sont décrites dans le tableau 3. Certaines soulèvent des questions soit en raison d'un conflit personnel entre le traducteur et Darwin (le cas de Royer), soit en raison de la méthode de traduction employée (le cas de Becquemont; voir plus bas). Certaines éditions anglaises n'ont pas été traduites (cases hachurées). 
TABleau 2

Tableau comparatif des différentes éditions anglaises de l'OS ${ }^{18}$

\begin{tabular}{|l|c|c|c|c|c|c|c|}
\hline Titre & \multicolumn{6}{|c|}{$\begin{array}{l}\text { On the origin of species by means of natural selection, } \\
\text { or the preservation of favoured races in the struggle } \\
\text { for life }\end{array}$} & $\begin{array}{c}\text { The origin of species by means of } \\
\text { natural selection, or the } \\
\text { preservation of favoured races in the } \\
\text { struggle for life }\end{array}$ \\
\hline $\begin{array}{l}\text { Année de } \\
\text { parution }\end{array}$ & 1859 & 1860 & 1861 & 1866 & 1869 & 1872 & 1876 \\
\hline Édition & 1 & $2^{19}$ & 3 & $4{ }^{20}$ & 5 & $6 a^{21}$ & $6 \mathrm{~b}$ \\
\hline $\begin{array}{l}\text { Nombre de } \\
\text { chapitres }\end{array}$ & & & 14 & & & & 15 \\
\hline $\begin{array}{l}\text { Nombre } \\
\text { de pages }\end{array}$ & 490 & 490 & 525 & 577 & 579 & 429 & 429 \\
\hline $\begin{array}{l}\text { Nombre } \\
\text { de mots* }\end{array}$ & 150190 & 150885 & 159938 & 176108 & 175632 & 192283 & 192283 \\
\hline Code & EN1859 & EN1860 & EN1861 & EN1866 & EN1869 & EN1872 & EN1876 \\
\hline
\end{tabular}

* Corps du texte seulement

TABLEAU 3

Tableau comparatif des traductions françaises de l'OS ${ }^{22}$

\begin{tabular}{|c|c|c|c|c|}
\hline Année EN & Édition anglaise & Code & Année FR & Traducteur \\
\hline \multirow{2}{*}{1859} & \multirow{2}{*}{1} & FR2008 & $1992 / 2008^{23}$ & Daniel Becquemont \\
\hline & & FR2013 & $2013^{24}$ & Thierry Hoquet \\
\hline 1860 & 2 & & & \\
\hline \multirow{5}{*}{1861} & \multirow{5}{*}{3} & FR1862 & 1862 & \multirow{5}{*}{ Clémence-Auguste Royer } \\
\hline & & FR1866 & $1866^{25}$ & \\
\hline & & FR1870 & $1870^{26}$ & \\
\hline & & FR1883 & $1883 ?^{27}$ & \\
\hline & & FRsd & s.d. ${ }^{28}$ & \\
\hline 1866 & 4 & & & \\
\hline 1869 & 5 & FR1873 & 1873 & Jean-Jacques Moulinié \\
\hline 1872 & $6 a$ & FR1876 & 1876 & Edmond Barbier \\
\hline 1876 & $6 \mathrm{~b}$ & FR2009 & 2009 & Aurélien Berra \\
\hline
\end{tabular}

Année EN ou FR: année de parution de l'édition anglaise ou de la traduction française

Les tableaux 2 et 3 montrent que les différentes versions de l'OS et leurs traductions totalisent plus de deux millions de mots, ce qui exclut d'emblée une annotation de l'ensemble. Le critère de faisabilité de l'étude a donc imposé une délimitation raisonnée du corpus à étudier s'appuyant sur des critères de validité formulés à partir d'une analyse de la structure de l'œuvre et de ses traductions. Premièrement, nous avons choisi de limiter notre étude au quatrième chapitre de l'ouvrage, intitulé «Natural Selection» ("Natural Selection; or Survival of the Fittest», dans les éditions de 1869 et 1872/1876), qui traite du concept-clé de la TE darwinienne: la sélection naturelle comme moteur de l'évolution des espèces. Avec le chapitre V, qui traite des lois de la variation, il s'agit du chapitre le plus important de l'OS (Hoquet 2013: 164). Bien qu'indépendant, notre choix s'est révélé identique à celui de Vandepitte, Vandenbussche, et al. (2011). 
Deuxièmement, nous avons retenu quatre des dix traductions françaises de l'OS présentées dans le tableau 3: FR1862 (Royer), FR1873 (Moulinié), FR1876 (Barbier) et FR2009 (Berra). Dans le cas de Royer, nous n'avons retenu qu'une seule des cinq traductions, celle de 1862, soit la première (FR1862). Une comparaison informatique des textes en format électronique, réalisée à l'aide de la fonction Comparaison de textes dans MS Word, a montré que les traductions de 1862, 1866 et 1870 étaient pratiquement identiques ${ }^{29}$. Mis à part quelques changements de typographie et de ponctuation, la seule modification notable est un changement terminologique: «élection naturelle» (1862), choix terminologique critiqué entre autres par Conry, devient «sélection naturelle» $(1866 ; 1870)$. Pour sa part, la traduction de Hoquet n'est parue qu'en mars 2013, bien après que notre recherche ait débuté; nous n'avons donc pas pu la prendre en compte. Quant à la traduction de Becquemont (2008), elle se démarque par sa stratégie inusitée: Becquemont a travaillé à partir de la traduction de la $6^{\mathrm{e}}$ édition anglaise par Edmond Barbier dans le but d'aboutir à une traduction de la $1^{\text {re }}$ édition anglaise ${ }^{30}$. Cette traduction a été écartée, car elle fera l'objet d'une étude à part.

Troisièmement, il a fallu régler un problème lié à la comparaison de traductions issues, respectivement, de textes sources successifs et donc présentant des différences: EN1861, EN1869, EN1872 et EN1876. En effet, prendre en considération ces quatre éditions anglaises dans leur intégralité ne permet pas de comparer les traductions entre elles. Pour cela, il faut un texte source unique. Nous avons donc repéré, dans le chapitre IV, les phrases qui sont demeurées identiques, ou qui n'ont été que très légèrement modifiées (par exemple, des modifications de ponctuation ou de typographie, le remplacement d'un mot unique, un changement dans l'ordre des mots, des reformulations superficielles) entre les différentes éditions de l'OS. Ceci a pu être réalisé grâce à l'outil variorum accessible gratuitement sur le site Web The Complete Works of Charles Darwin Online ${ }^{31}$, sous la direction de John van Wyhe. Nous avons ainsi pu identifier 311 phrases totalisant 11260 mots.

Finalement, la faisabilité de l'annotation en elle-même s'est révélée une contrainte importante dans la stratégie adoptée, ce qui a nécessité, aux fins de la comparaison des traductions, de restreindre encore plus le corpus de 311 phrases décrit précédemment ( 311 phrases $\times 5$ textes $=1555$ phrases, soit un minimum de 56300 mots). La stratégie d'annotation de corpus numérisés que nous allons décrire plus loin autorise en effet une grande finesse et une systématicité remarquable. En contrepartie, elle est chronophage. De plus, elle constitue un défi particulier sur le plan informatique en raison de l'usage d'un corpus parallèle comportant plusieurs traductions (Gendron-Pontbriand, Bodain, et al. 2016). Nous avons donc tiré parti d'une étude parallèle (Gendron-Pontbriand 2013) qui nous avait permis de cibler des segments enrichis en conceptualisation métaphorique, permettant notamment à Darwin de formuler sa théorie. Plusieurs auteurs ont souligné le rôle crucial des métaphores dans la théorisation de la biologie (voir par exemple Vandaele 2009), particulièrement dans l'OS (Young 1985; Ruse 2000). Les phrases où la métaphorisation est dense représentent donc des segments riches conceptuellement. Procéder à l'analyse de la modalité épistémique sur ces segments nous permettra d'examiner ultérieurement des hypothèses de corrélation entre ces phénomènes. Un total de 55 phrases riches en conceptualisation métaphorique, totalisant 2645 mots, avaient été isolées parmi les 311 phrases sélectionnées. Les quatre traductions de ces 55 phrases constituent 
la portion française de notre corpus. Elles totalisent respectivement 2964 (FR1862), 2728 (FR1873), 2879 (FR1876) et 2946 (FR2009) mots. La composition et la taille totale de notre corpus sont décrites dans la figure 2 .

FIGURE 2

Composition et taille du corpus ${ }^{32}$

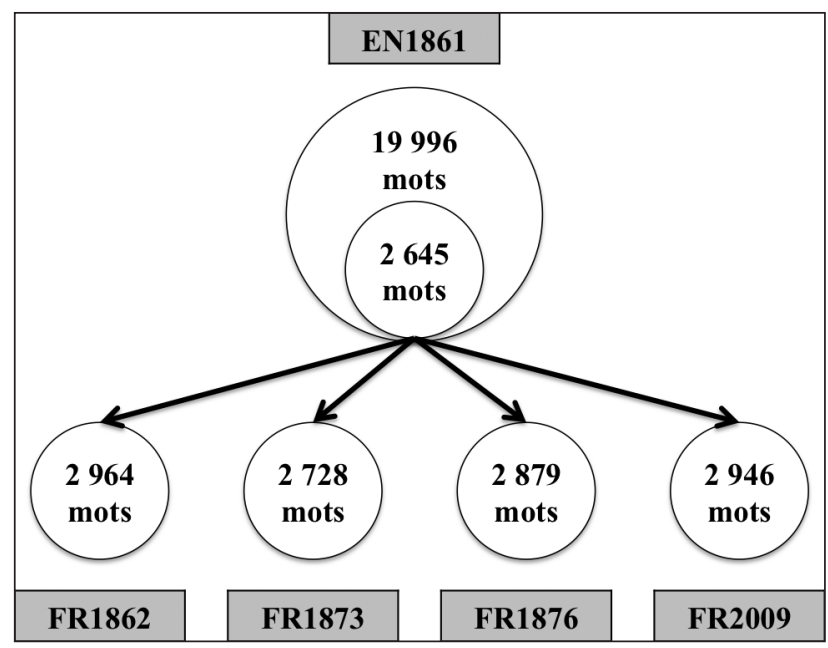

\subsection{Annotation manuelle de texte dans l'environnement $X M L$}

Hyland note que la MEA dépend du contexte: «[...] the interpretation of hedges in RAs cannot be captured by assigning particular semantic meanings to decontextualised forms: particular devices can only be interpreted as conveying hedging meaning in the utterance of actual sentences» (Hyland 1999: 155). Pour éviter une circularité qui découlerait de l'usage automatisé d'une liste prédéfinie, il a été nécessaire d'adopter une stratégie reposant sur le repérage «manuel» des MMEA en corpus. Pour ce faire, nous avons adapté la méthode d'annotation sémantique de textes décrite dans Gendron-Pontbriand, Bodain, et Vandaele (2016) pour le repérage des métaphores conceptuelles $^{33}$. Réalisée grâce au langage de balisage XML et à certains de ses dérivés (XQuery, XSLT) et à l'aide du logiciel Oxygen XML Editor 14.0, elle permet de repérer et de caractériser les MMEA directement dans le corpus, converti en format $\mathrm{XML}$, et ce, en les entourant de balises. Une balise est un élément de langage XML délimité par deux chevrons $(<\ldots>)$. Les éléments balisés doivent être précédés d'une balise ouvrante et suivis d'une balise fermante. Des attributs insérés dans la balise permettent de consigner des informations linguistiques relatives à l'élément balisé. Le recours à l'annotation in situ à l'aide du langage XML comporte de nombreux avantages : notamment, outre sa précision, elle donne au chercheur une grande flexibilité dans le choix des attributs et lui permet de les mettre à jour facilement.

Dans le présent projet, nous avons utilisé une seule balise, nommée $<$ hedg $>$, dont les cinq attributs sont décrits dans le tableau 4. 
TABLEAU 4

Description de la balise $<$ hedg $>$

\begin{tabular}{|l|l|l|}
\hline \multicolumn{2}{|l|}{ hedg $>$} \\
\hline Attribut & Contenu & Description \\
\hline id & Chiffre & Numéro unique \\
\hline lex & Texte & Catégorie du discours à laquelle appartient le MMEA annoté \\
\hline lem & Texte & Forme lemmatisée du MMEA annoté \\
\hline nat & Texte & Type de MMEA \\
\hline comm & Texte & Commentaires \\
\hline
\end{tabular}

L'attribut @id permet d'attribuer à chaque élément un numéro d'identification unique. Les attributs @lex (abréviation de «lexical») et @lemme permettent, respectivement, de spécifier la catégorie du discours à laquelle appartient l'élément annoté et sa forme lemmatisée, afin de permettre l'extraction de données en fonction de ces paramètres. L'attribut @comm permet à l'annotateur d'insérer des commentaires dans le texte. Finalement, l'attribut @nat (abréviation de "nature») nous permet de spécifier le type de MMEA à partir d'une liste de valeurs qui apparaît dans un menu déroulant de l'interface d'annotation. Cette liste découle d'une adaptation de la typologie des MMEA établie par Hyland (1999), présentée dans le tableau 5.

\section{TABleau 5}

Valeurs possibles de l'attribut @nat

\begin{tabular}{|c|c|c|c|}
\hline & & Valeurs & Exemples \\
\hline 1 & \multirow{5}{*}{$\begin{array}{l}\text { MMEA portant sur } \\
\text { la factivité de la } \\
\text { proposition (la } \\
\text { proposition est-elle } \\
\text { vraie ou fausse?) }\end{array}$} & $\begin{array}{l}\text { Modalité inhérente, sans } \\
\text { les auxiliaires modaux }\end{array}$ & $\begin{array}{l}\text { "Thus it is, as I believe, that two or more genera } \\
\text { are produced }[. . .] »(\text { Darwin } 1861: 129)\end{array}$ \\
\hline 2 & & Auxiliaires modaux & $\begin{array}{l}\text { "In a large genus it is probable that more than } \\
\text { one species would vary." (Darwin } 1861: 126 \text { ) }\end{array}$ \\
\hline 3 & & Phrase interrogative & $\begin{array}{l}\text { "What reason, it may be asked, is there for } \\
\text { supposing in these cases that two individuals ever } \\
\text { concur in reproduction?" (Darwin 1861: 101) }\end{array}$ \\
\hline 4 & & Flexion verbale & $\begin{array}{l}{[\ldots] \text { dans ce cas, il y aurait une rétrogradation }} \\
\text { réelle dans l'échelle de l'organisation.» (Darwin } \\
\text { 1872/1876: 134, traduit par Barbier; voir note 11) }\end{array}$ \\
\hline 5 & & $\begin{array}{l}\text { Auxiliaire modal avec } \\
\text { flexion }\end{array}$ & $\begin{array}{l}\text { "D'autres modifications de la même nature } \\
\text { pourront encore accroître cet avantage [...]» } \\
\text { (Darwin } 1872 / 1876: 88 \text {, traduit par Barbier; voir } \\
\text { note 11) }\end{array}$ \\
\hline 6 & \multirow{3}{*}{$\begin{array}{l}\text { MMEA portant sur } \\
\text { la source du } \\
\text { jugement } \\
\text { épistémique (qui } \\
\text { émet le jugement?) }\end{array}$} & Tournure impersonnelle & $\begin{array}{l}\text { "In a large genus it is probable that more than } \\
\text { one species would vary." (Darwin } 1861: 126 \text { ) }\end{array}$ \\
\hline 7 & & $\begin{array}{l}\text { Tournure personnelle - } \\
1^{\text {re }} \text { personne du singulier }\end{array}$ & $\begin{array}{l}\text { "This preservation of favourable variations and } \\
\text { the rejection of injurious variations, I call Natural } \\
\text { Selection.» (Darwin 1861:84) }\end{array}$ \\
\hline 8 & & $\begin{array}{l}\text { Tournure personnelle } \\
-1^{\text {re }} \text { personne du pluriel }\end{array}$ & $\begin{array}{l}\text { "We may conclude, from what we have seen of } \\
\text { the intimate and complex manner }[\ldots] \text { ".. } \\
\text { (Darwin } 1861: 85 \text { ) }\end{array}$ \\
\hline
\end{tabular}

Les phrases interrogatives (ligne 3 du tableau 5) et les tournures impersonnelles (ligne 6) sont des éléments qui ont été repris intégralement du modèle de Hyland. Tous les MMEA lexicaux, c'est-à-dire tous ceux dont le caractère modal est inhérent à leur sens lexical, se voient attribuer la valeur «modalité inhérente» pour l'attribut @nat (ligne 1); l'attribut @lex permet de les catégoriser en fonction de la partie du 
discours à laquelle ils appartiennent. Ainsi, nous prenons en compte tous les types de MMEA lexicaux décrits par Hyland. Les auxiliaires modaux ont une valeur distincte pour les distinguer des verbes lexicaux (ligne 2).

Nous prenons également en compte certains éléments présents dans le modèle de Hyland, mais que celui-ci n'approfondit pas. D’une part, bon nombre de phrases contiennent des pronoms personnels à la première personne du singulier ou du pluriel (je/nous et I/we) (lignes 7 et 8 ) : il s'agit d'une marque claire de la subjectivité de l'auteur, et c'est à ce titre que ces pronoms ont été annotés. En effet, Hyland (1999: 45) affirme que l'usage de ces pronoms identifie explicitement l'auteur comme étant la source du jugement épistémique; ainsi la proposition n'apparaît pas comme une affirmation catégorique, mais comme l'opinion de l'auteur. Il est possible que ces pronoms personnels n'aient pas été abordés en détail par Hyland en raison de leur utilisation presque inexistante dans les articles de recherche scientifique modernes, qui constituaient l'objet de son étude. D'autre part, le modèle de Hyland étant construit à partir d'un corpus anglais, il ne rend pas compte des flexions verbales. Comme le souligne Larreya (2004: 741), les formes flexionnelles de sens modal existent en français, mais pas en anglais. Nous les avons donc prises en compte lors de l'annotation des traductions françaises de l'OS (lignes 4 et 5).

Par ailleurs, nous n'avons pas pris en compte les formes passives. En effet, tout en reconnaissant que les verbes à la voix passive permettent à l'auteur de se distancier de ses propres affirmations, Hyland (1999: 5) souligne que ces MMEA syntaxiques sont très hétérogènes et leur caractérisation est donc ardue. Lui-même affirme que les formes passives nécessitent une étude distincte; il ne les prend d'ailleurs pas en compte dans son analyse. Une caractérisation plus fine et plus complète des MMEA de forme passive est ainsi nécessaire avant que ceux-ci puissent faire l'objet d'analyse.

Enfin, pour évaluer statistiquement la représentativité de l'échantillon sélectionné, nous avons annoté la totalité du chapitre IV dans sa version de 1861 (première édition anglaise à avoir été traduite en français; voir note 2). Ceci nous a permis d'obtenir la densité et la répartition des MMEA dans l'ensemble du chapitre.

\subsection{Extraction et analyse des données}

Une fois les textes annotés, le langage de requête XQuery permet d'extraire les données brutes en fonction des valeurs consignées dans les attributs présents dans les balises. Ces données sont ensuite transférées dans un tableur Excel à des fins d'analyses qualitative et quantitative. Nous avons recours au test statistique du $\mathrm{X}^{2}$ (chicarré $^{34}$ afin, d'une part, d'évaluer la représentativité de l'échantillon de 55 phrases par rapport à l'ensemble du chapitre IV en anglais et, d'autre part, de comparer les traductions à leur texte source, mais aussi entre elles. Le test porte sur la comparaison des répartitions des MMEA dans les différentes catégories de modalité épistémique. L'interprétation des résultats du test a été réalisée à l'aide d'une table de $\mathrm{X}^{2}$ standard $^{35}$ et d'un calculateur de valeur $p^{36}$.

\section{Résultats}

Après l'évaluation de la représentativité de l'échantillon de 55 phrases dans la langue source, nous présenterons le nombre et la répartition des MMEA relevés dans le texte 
anglais et dans chacune des traductions françaises, ce qui nous permettra de comparer chacune des traductions avec le texte anglais, mais aussi entre elles. Finalement, nous déterminerons s'il existe un changement qualitatif de la modalité épistémique entre le texte anglais et les différentes traductions.

\subsection{Caractérisation de l'échantillon de 55 phrases}

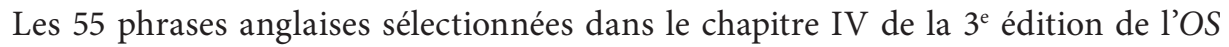
(EN1861) selon la méthode décrite à la section 5.1 constituent le pivot de l'analyse. Elles constituent 12,5\% du chapitre. Dans un premier temps, nous avons évalué leur représentativité par rapport à l'ensemble du chapitre en ce qui concerne la répartition des différents types de MMEA, et ce, à l'aide d'un test du $\mathrm{X}^{2}$. Pour ce faire, nous avons appliqué la première méthode utilisée dans Gendron-Pontbriand, Bodain, et al. (2016: 726) et adaptée de Müller (1968: 95-96). Ainsi, nous avons calculé la distribution théorique en ramenant la distribution marginale à l'effectif observé pour le chapitre complet. Cette technique permet donc la comparaison d'une partie d'un corpus avec le corpus lui-même. Le tableau 6 montre le nombre d'occurrences de tous les MMEA annotés dans le texte source (EN1861) et dans les 55 phrases de notre échantillon (55 Phr), ainsi que le rapport entre les occurrences présentes dans l'échantillon et dans le chapitre complet, exprimé en pourcentage.

TABLEAu 6

Nombre d'occurrences des MMEA en fonction de leur typologie

\begin{tabular}{|c|c|c|c|}
\hline Types de MMEA & EN1861 & $55 \mathrm{Phr}$ & \% d'EN1861 \\
\hline Modalité inhérente & 296 & 47 & 15,9 \\
\hline Auxiliaires modaux & 526 & 88 & 16,7 \\
\hline Tournures personnelles - $1^{\text {re }}$ personne du singulier & 127 & 10 & 7,9 \\
\hline Tournures personnelles $-1^{\text {re }}$ personne du pluriel & 157 & 17 & 10,8 \\
\hline Tournures impersonnelles & 104 & 12 & 11,5 \\
\hline Phrases interrogatives & 30 & 2 & 6,7 \\
\hline TOTAL & 1248 & 176 & 14,1 \\
\hline
\end{tabular}

Le tableau 6 montre que, pour la majorité des catégories, l'échantillon comporte entre $7 \%$ et $20 \%$ des occurrences de MMEA alors qu'il représente $12,5 \%$ de l'ensemble du chapitre. Pour les besoins du calcul du $\mathrm{X}^{2}$, les phrases interrogatives, dont l'effectif est faible, ont été regroupées avec les tournures impersonnelles ${ }^{37}$. La valeur du $\mathrm{X}^{2}$ pour les données du tableau 6 est donc de 93,34 pour un degré de liberté de 4, ce qui correspond à une valeur $p$ de 0,00000103. En d'autres mots, cela signifie que l'on a une probabilité de $0,000103 \%$ de se tromper en affirmant que les deux distributions sont différentes, ce qui est en deçà du seuil généralement admis de $5 \%$. La sélection non aléatoire de l'échantillon (sur la base de la présence d'expressions métaphoriques, voir section 5.1) a donc une incidence sur la distribution des MMEA. Il est logique de penser que, dans les passages où Darwin établit les assises conceptuelles de sa théorie, la densité des MMEA est différente de celle du reste du texte. Nous pouvons néanmoins conclure que l'échantillon est valide pour les besoins de 
notre étude, dans la mesure où tous les types de MMEA recensés dans l'ensemble du chapitre s'y trouvent également, et ce, avec un effectif suffisant pour permettre l'application du test du $\mathrm{X}^{2}$ (exception faite des phrases interrogatives). Étant donné le petit nombre de phrases interrogatives dans l'échantillon, les conclusions relatives à cette catégorie devront être prudentes.

\subsection{Répartition des MMEA dans les différentes traductions françaises de l'OS}

\subsubsection{Répartition globale}

Les résultats décrits dans la suite de l'article sont tirés exclusivement de l'échantillon de 55 phrases en anglais et de ses quatre traductions en français. Le tableau 7 présente le nombre d'occurrences des MMEA dans les cinq échantillons. Nous avons comparé la répartition des MMEA dans l'échantillon anglais et dans chacune des traductions à l'aide du test du $\mathrm{X}^{2}$, cette fois-ci en appliquant la deuxième méthode proposée par Gendron-Pontbriand, Bodain, et al. (2016). Selon cette méthode, $\mathrm{H}_{0}$ correspond à une hypothèse d'équivalence parfaite: les répartitions des MMEA dans l'original anglais et dans la traduction française seraient identiques. La distribution théorique correspond donc à la répartition des MMEA dans l'échantillon anglais.

À nouveau, les phrases interrogatives ont été regroupées avec les tournures impersonnelles en raison de leur faible effectif. Il faut également noter que l'anglais et le français ont des moyens grammaticaux spécifiques pour exprimer la modalité. Or, le test du $\mathrm{X}^{2}$ exige des catégories cohérentes entre les distributions à l'étude. Pour autoriser l'usage du test, nous faisons donc correspondre à la catégorie homogène des auxiliaires modaux en anglais (p. ex. : may, can, etc.) une catégorie grammaticalement hétérogène en français, mais homogène sur le plan du sens, constituée d'auxiliaires modaux fléchis (p. ex.: pourrait, devrait, etc.) ou non (p. ex.: doit, peut, etc.), ainsi que de verbes lexicaux au conditionnel (p. ex.: semblerait, etc.) ou au futur quand ce dernier exprime une atténuation (p. ex.: verrons, etc.).

TABLEAU 7

Nombre d'occurrences de chaque type de MMEA dans l'échantillon anglais et dans chacune des traductions françaises

\begin{tabular}{|l|c|c|c|c|c|}
\hline Types de MMEA & $\begin{array}{c}\text { EN1861 } \\
\text { Darwin }\end{array}$ & $\begin{array}{c}\text { FR1862 } \\
\text { Royer }\end{array}$ & $\begin{array}{c}\text { FR1873 } \\
\text { Moulinié }\end{array}$ & $\begin{array}{c}\text { FR1876 } \\
\text { Barbier }\end{array}$ & $\begin{array}{c}\text { FR2009 } \\
\text { Berra }\end{array}$ \\
\hline Modalité inhérente & 47 & 40 & 44 & 43 & 44 \\
\hline Auxiliaires modaux et flexions & 88 & 63 & 72 & 57 & 72 \\
\hline Tournures personnelles $-1^{\text {re }}$ personne du singulier & 10 & 7 & 13 & 9 & 10 \\
\hline Tournures personnelles $-1^{\text {re }}$ personne du pluriel & 17 & 8 & 12 & 12 & 16 \\
\hline Tournures impersonnelles & 12 & 25 & 17 & 31 & 20 \\
\hline Phrases interrogatives & 2 & 2 & 2 & 2 & 1 \\
\hline TOTAL & $\mathbf{1 7 6}$ & $\mathbf{1 4 5}$ & $\mathbf{1 6 0}$ & $\mathbf{1 5 4}$ & $\mathbf{1 6 3}$ \\
\hline & $\mathbf{X}^{2}$ & 25,88 & 7,26 & 38,32 & 6,66 \\
\hline
\end{tabular}


La première observation est qu'aucune des traductions françaises ne comporte le même nombre de MMEA que l'échantillon anglais. La traduction de Berra est celle qui comporte le plus de MMEA, alors que celle de Royer en comporte le moins. Ensuite, les résultats du test du $\mathrm{X}^{2}$ montrent que les traductions de Royer et de Barbier sont significativement différentes du texte anglais (valeurs $p$ inférieures à $\alpha=0,05$ ) sur le plan de la répartition des MMEA. En effet, la probabilité de se tromper en affirmant que chacune de ces traductions est significativement différente du texte anglais est inférieure à $0,01 \%$. En revanche, les répartitions des MMEA dans les traductions de Moulinié et de Berra ne sont pas significativement différentes de l'original $(p>0,1)$.

\subsubsection{Influence $d u$ processus de traduction}

Le test du $\mathrm{X}^{2}$ effectué sur les données du tableau 7 offre une vision globale de la répartition des MMEA dans l'original anglais et les quatre traductions françaises. Cependant, il ne reflète pas la réalité du processus de traduction. En l'occurrence, les MMEA présents dans le texte original peuvent être traduits ou omis dans le texte cible; certains MMEA peuvent également avoir été ajoutés dans la traduction. Le tableau 8 montre le nombre de MMEA omis et ajoutés dans chacune des traductions en fonction de leur type.

TABLEAu 8

Nombre de MMEA anglais omis et nombre de MMEA ajoutés dans chacune des traductions françaises en fonction de leurs types

\begin{tabular}{|l|c|c|c|c|c|c|c|c|}
\hline \multirow{2}{*}{ Types de MMEA } & \multicolumn{2}{|c|}{$\begin{array}{c}\text { FR1862 } \\
\text { Royer }\end{array}$} & \multicolumn{2}{c|}{$\begin{array}{c}\text { FR1873 } \\
\text { Moulinié }\end{array}$} & \multicolumn{2}{c|}{$\begin{array}{c}\text { FR1876 } \\
\text { Barbier }\end{array}$} & \multicolumn{2}{c|}{$\begin{array}{c}\text { FR2009 } \\
\text { Berra }\end{array}$} \\
\cline { 2 - 10 } & O & A & O & A & O & A & O & A \\
\hline Modalité inhérente & 12 & 6 & 8 & 5 & 9 & 6 & 4 & 1 \\
\hline Auxiliaires modaux et flexions & 29 & 2 & 26 & 10 & 37 & 5 & 16 & 0 \\
\hline Tournures personnelles $-1^{\text {re }}$ personne du singulier & 0 & 0 & 0 & 1 & 1 & 0 & 1 & 0 \\
\hline Tournures personnelles $-1^{\text {re }}$ personne du pluriel & 5 & 2 & 2 & 2 & 1 & 1 & 0 & 0 \\
\hline Tournures impersonnelles & 6 & 11 & 5 & 7 & 5 & 19 & 2 & 10 \\
\hline Phrases interrogatives & 0 & 0 & 0 & 0 & 0 & 0 & 1 & 0 \\
\hline \multicolumn{1}{c}{ TOTAL } & $\mathbf{5 2}$ & $\mathbf{2 1}$ & $\mathbf{4 1}$ & $\mathbf{2 5}$ & $\mathbf{5 3}$ & $\mathbf{3 1}$ & $\mathbf{2 4}$ & $\mathbf{1 1}$ \\
\hline
\end{tabular}

$\mathrm{O}=$ omission $; \mathrm{A}=$ ajout

On observe que la traduction de Barbier comporte le plus d'omissions (53) ce qui correspond à $30 \%$ de tous les MMEA repérés dans l'échantillon anglais (176). La traduction de Royer comporte presque autant d'omissions (52) que celle de Barbier. La traduction de Berra, au contraire, contient le moins d'omissions (25), ce qui représente $14 \%$ des MMEA relevés dans l'original. Parmi les omissions, ce sont les auxiliaires modaux qui en font le plus les frais: 29/52 (56\%) chez Royer, 26/41 (63\%) chez Moulinié, 37/53 (70\%) chez Barbier et 16/24 (67\%) chez Berra. L'expression de la modalité inhérente suit, avec 12/52 (23\%) pour Royer, 8/41 (20\%) pour Moulinié, 9/41 (22\%) pour Barbier et 4/24 (17\%) pour Berra. En ce qui concerne les MMEA ajoutés, la traduction de Barbier est celle qui en contient le plus, alors que la traduction 
de Berra en comporte le moins. Parmi les ajouts, ce sont les tournures impersonnelles qui sont en général le plus représentées : 11/21 (52\%) chez Royer, 19/31 (61\%) chez Barbier et 10/11 (91\%) chez Berra. Dans le cas de la traduction de Moulinié, ce sont les ajouts d'auxiliaires modaux qui sont les plus fréquents $(10 / 25=40 \%)$.

En plus des MMEA omis ou ajoutés, notre méthode d'annotation permet aussi de comptabiliser, pour chaque traduction, tous les MMEA traduits en fonction du type de transfert: le MMEA traduit est du même type que le MMEA original anglais ou le MMEA traduit est d'un autre type. Ces données, ainsi que les ajouts et les omissions, sont présentées dans le tableau 9.

TABLEAU 9

Nombre d'occurrences de chaque type de MMEA dans chacune des traductions françaises en fonction du type de transfert

\begin{tabular}{|c|c|c|c|c|c|c|c|}
\hline \multicolumn{4}{|c|}{ Catégories de transfert } & \multirow{2}{*}{$\begin{array}{c}\text { FR1862 } \\
\text { Royer }\end{array}$} & \multirow{2}{*}{$\begin{array}{c}\text { FR1873 } \\
\text { Moulinié } \\
38\end{array}$} & \multirow{2}{*}{$\begin{array}{c}\text { FR1876 } \\
\text { Barbier } \\
37\end{array}$} & \multirow{2}{*}{$\begin{array}{c}\text { FR2009 } \\
\text { Berra }\end{array}$} \\
\hline 1 & Modalité inhérente & $\rightarrow$ & Modalité inhérente & & & & \\
\hline 2 & Modalité inhérente & $\rightarrow$ & Aux. modaux et flexions & 0 & 1 & 1 & 0 \\
\hline 3 & $\varnothing$ & $\rightarrow$ & Modalité inhérente & 6 & 5 & 6 & 1 \\
\hline 4 & Modalité inhérente & $\rightarrow$ & $\varnothing$ & 12 & 8 & 9 & 4 \\
\hline 5 & Aux. modaux & $\rightarrow$ & Aux. modaux et flexions & 60 & 61 & 51 & 72 \\
\hline 6 & Aux. modaux & $\rightarrow$ & Modalité inhérente & 0 & 1 & 0 & 0 \\
\hline 7 & $\varnothing$ & $\rightarrow$ & Aux. modaux et flexions & 2 & 10 & 5 & 0 \\
\hline 8 & Aux. modaux & $\rightarrow$ & $\varnothing$ & 29 & 26 & 37 & 16 \\
\hline 9 & T. pers. $1^{\text {re }}$ p. sing. & $\rightarrow$ & T. pers. $1^{\text {re }}$ p. sing. & 7 & 9 & 8 & 9 \\
\hline 10 & T. pers. $1^{\text {re }}$ p. sing. & $\rightarrow$ & T. pers. $1^{\text {re }}$ p. pl. & 1 & 0 & 0 & 0 \\
\hline 11 & T. pers. $1^{\text {re }}$ p. sing. & $\rightarrow$ & T. impersonnelles & 1 & 1 & 1 & 0 \\
\hline 12 & T. pers. $1^{\text {re }}$ p. sing. & $\rightarrow$ & Aux. modaux et flexions & 1 & 0 & 0 & 0 \\
\hline 13 & $\varnothing$ & $\rightarrow$ & T. pers. $1^{\text {re }}$ p. sing. & 0 & 1 & 0 & 0 \\
\hline 14 & T. pers. $1^{\text {re }}$ p. sing. & $\rightarrow$ & $\varnothing$ & 0 & 0 & 1 & 1 \\
\hline 15 & T. pers. $1^{\mathrm{re}} \mathrm{p}$. pl. & $\rightarrow$ & T. pers. $1^{\text {re }}$ p. pl. & 5 & 10 & 10 & 16 \\
\hline 16 & T. pers. $1^{\mathrm{re}} \mathrm{p}$. pl. & $\rightarrow$ & T. impersonnelles & 7 & 2 & 4 & 0 \\
\hline 17 & T. pers. $1^{\text {re }} \mathrm{p}$. pl. & $\rightarrow$ & T. pers. $1^{\text {re }}$ p. sing & 0 & 3 & 1 & 1 \\
\hline 18 & $\varnothing$ & $\rightarrow$ & T. pers. $1^{\text {re }}$ p. pl. & 2 & 2 & 1 & 0 \\
\hline 19 & T. pers. $1^{\mathrm{re}} \mathrm{p}$. pl. & $\rightarrow$ & $\varnothing$ & 5 & 2 & 1 & 0 \\
\hline 20 & T. impersonnelles & $\rightarrow$ & T. impersonnelles & 6 & 7 & 7 & 10 \\
\hline 21 & T. impersonnelles & $\rightarrow$ & T. pers. $1^{\mathrm{re}} \mathrm{p} . \mathrm{pl}$. & 0 & 0 & 1 & 0 \\
\hline 22 & $\varnothing$ & $\rightarrow$ & T. impersonnelles & 11 & 7 & 19 & 10 \\
\hline 23 & T. impersonnelles & $\rightarrow$ & $\varnothing$ & 6 & 5 & 5 & 2 \\
\hline 24 & Phrases interrogatives & $\rightarrow$ & Phrases interrogatives & 2 & 2 & 2 & 1 \\
\hline 25 & $\varnothing$ & $\rightarrow$ & Phrases interrogatives & 0 & 0 & 0 & 0 \\
\hline 26 & Phrases interrogatives & $\rightarrow$ & $\varnothing$ & 0 & 0 & 0 & 1 \\
\hline
\end{tabular}

Afin d'autoriser l'application du test du $\mathrm{X}^{2}$ (nécessité d'avoir des effectifs supérieurs à 5 dans chaque catégorie), nous avons regroupé certaines catégories du tableau 9. Nous avons effectué les regroupements suivants: les MMEA ajoutés dans le texte cible $(3,7,13,18,22$ et 25); les MMEA de modalité inhérente en anglais modifiés ou omis en français (2 et 4); les auxiliaires modaux en anglais modifiés ou omis en français (6 et 8); les tournures personnelles à la $1^{\text {re }}$ personne du singulier et 
à la $1^{\text {re }}$ personne du pluriel en anglais modifiées ou omises en français $(10,11,12,14$, 16, 17 et 19); les tournures impersonnelles et les phrases interrogatives ( 20 et 24 ; voir note 37 ); les tournures impersonnelles et les phrases interrogatives modifiées ou omises en français $(21,23$ et 26). Le tableau 10 montre le résultat de ce regroupement.

TABleau 10

Regroupement des données du tableau 9 pour le calcul du $\mathrm{X}^{2}$

\begin{tabular}{|c|c|c|c|c|c|c|}
\hline Catégories de transfert & & & $\begin{array}{c}\text { FR1862 } \\
\text { Royer }\end{array}$ & $\begin{array}{c}\text { FR1873 } \\
\text { Moulinié }\end{array}$ & $\begin{array}{l}\text { FR1876 } \\
\text { Barbier }\end{array}$ & $\begin{array}{c}\text { FR2009 } \\
\text { Berra }\end{array}$ \\
\hline Modalité inhérente & $\rightarrow$ & Modalité inhérente & 34 & 38 & 37 & 43 \\
\hline $\begin{array}{l}\text { Modalité inhérente / } \\
\text { T. pers. } 1^{\text {re }} \text { p. sing. / } \\
\text { T. pers. } 1^{\text {re }} \text { p. pl. / } \\
\text { T. impersonnelles / } \\
\text { P. interrogatives }\end{array}$ & $\rightarrow$ & $\varnothing$ ou $^{*}$ & 33 & 22 & 24 & 9 \\
\hline Aux. modaux & $\rightarrow$ & Aux. modaux et flexion & 60 & 61 & 51 & 72 \\
\hline Aux. modaux & $\rightarrow$ & $\varnothing \mathrm{ou}^{*}$ & 29 & 27 & 37 & 16 \\
\hline T. pers. $1^{\mathrm{re}}$ p. sing. & $\rightarrow$ & T. pers. $1^{\text {re }}$ p. sing. & 7 & 9 & 8 & 9 \\
\hline T. pers. $1^{\mathrm{re}}$ p. pl. & $\rightarrow$ & T. pers. $1^{\mathrm{re}} \mathrm{p} . \mathrm{pl}$ & 5 & 10 & 10 & 16 \\
\hline $\begin{array}{l}\text { T. impersonnelles / } \\
\text { P. interrogatives }\end{array}$ & $\rightarrow$ & $\begin{array}{l}\text { T. impersonnelles / } \\
\text { P. interrogatives }\end{array}$ & 8 & 9 & 9 & 11 \\
\hline$\varnothing$ & $\rightarrow$ & * & 21 & 25 & 31 & 11 \\
\hline
\end{tabular}

* = tout type de MMEA

Nous avons appliqué le test du $\mathrm{X}^{2}$ aux données du tableau 10 en comparant les traductions deux à deux. Ce faisant, nous comparons les stratégies de traduction des quatre traducteurs. La distribution théorique n'est ici pas connue. Nous avons donc adopté la méthode d'estimation des effectifs théoriques par tableau de contingence (Müller 1968: 96; Chanquoy 2005: 249-251). Les valeurs du $\mathrm{X}^{2}$ résultantes et les valeurs $p$ correspondantes sont présentées dans le tableau 11.

TABLEAU 11

Valeurs du $\mathrm{X}^{2}$ et valeurs $\mathrm{p}$ calculées sur les données du tableau 9

\begin{tabular}{|l|c|c|c|}
\hline Traductions & FR1873 (Moulinié) & FR1876 (Barbier) & FR2009 (Berra) \\
\hline FR1862 (Royer) & 4,79 & 6,72 & 28,98 \\
& $p=0,69$ & $p=0,46$ & $p=0,00015$ \\
\hline FR1873 (Moulinié) & & 3,17 & 16,02 \\
& & $p=0,87$ & $p=0,025$ \\
\hline FR1876 (Barbier) & & & 29,40 \\
& & & $p=0,00012$ \\
\hline
\end{tabular}

On constate que les stratégies de traduction de Berra (FR2009) sont significativement différentes de celles de toutes les autres traductions. En effet, pour chaque comparaison, la probabilité de se tromper en affirmant que la traduction de Berra est différente des autres traductions sur ce plan est inférieure à 1\%. Quant aux traductions de Royer (FR1862), de Moulinié (FR1873) et de Barbier (FR1876), on n'observe aucune différence significative entre elles $(p>0,05)$ sur le plan des stratégies de 
traduction. En effet, la probabilité de se tromper en affirmant que les stratégies de traduction sont différentes entre celles-ci varie entre $31 \%$ et $66 \%$.

Comme nous le mentionnons à la note 34 , la valeur du $\mathrm{X}^{2}$ et la valeur $p$ correspondante constituent une mesure de l'écart entre deux distributions. En plaçant les valeurs de $\mathrm{X}^{2}$ obtenues au tableau 11 sur un graphique ${ }^{38}$, on peut illustrer la distance relative entre les quatre traductions (voir figure 3 ).

FIGURE 3

Distance relative entre les traductions françaises par rapport à la répartition des différents types de MMEA

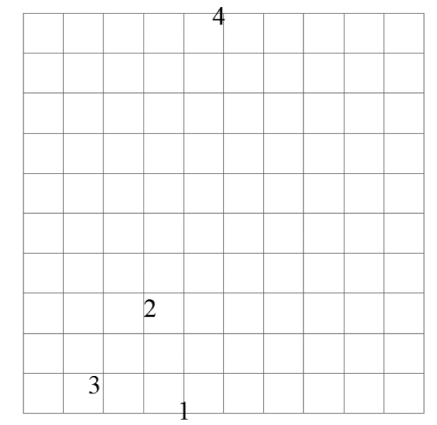

$1=$ Royer, 2 = Moulinié, 3 = Barbier, $4=$ Berra

\subsection{Effets de la traduction sur le degré de certitude}

Notre méthode d'extraction de données permet de créer des tableaux mettant en correspondance le texte source et chaque traduction française. Ainsi, il est possible de comparer la répartition des MMEA dans les textes source et cible, et de poser un jugement par rapport au changement de l'expression de la modalité épistémique, et ce, en se basant sur ce que nous avons appelé un « continuum de certitude», à l'instar de Desclés (2003: 55), en nous inspirant de la définition de la modalité épistémique proposée par Vilha $(1999: 20):$ «[...] it can be seen as a scale ranging from impossible through unlikely, possible, and probable to certain. " La figure 4, un histogramme empilé ramené à $100 \%$, présente les MMEA relevés dans chacune des traductions en fonction de leur effet sur la modalité épistémique: 1) «égal», le MMEA français se trouve au même endroit sur le continuum de certitude que son équivalent anglais; 2) «moins», le MMEA français rend le propos moins certain que son équivalent anglais; 3) «plus», le MMEA français rend le propos plus certain que son équivalent anglais. La répartition des MMEA dans chacune de ces catégories d'effet de modalité est exprimée en pourcentage; les valeurs absolues sont également indiquées. Nous présentons en annexe un échantillon du corpus d'analyse illustrant ces trois types d'effet sur la modalité épistémique. 


\section{FIGURE 4}

Effet de la traduction sur le degré de certitude des MMEA

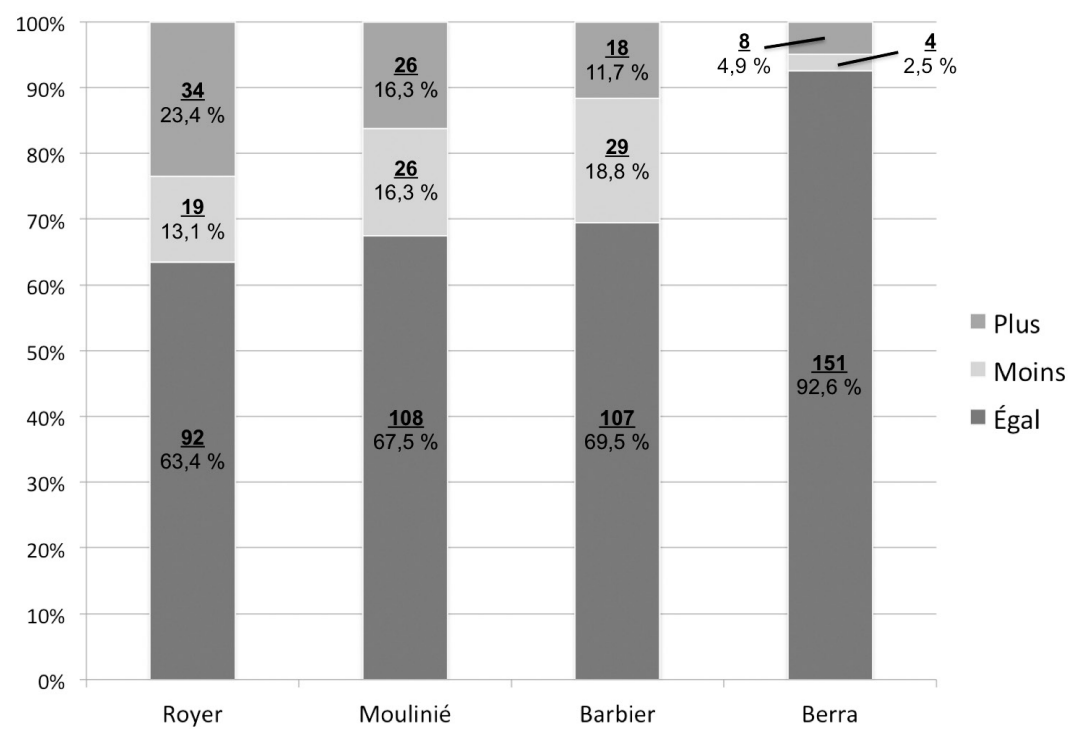

La figure 4 révèle que près de $93 \%$ des MMEA repérés dans la traduction de Berra expriment le même degré de certitude que les MMEA correspondants dans l'échantillon anglais, ce qui en fait la traduction la plus équivalente à l'échantillon anglais sur le plan du degré de certitude global exprimé dans le texte. À l'opposé, la traduction de Royer s'éloigne le plus de l'échantillon anglais; en effet, $63 \%$ des MMEA repérés dans la traduction de Royer expriment le même degré de certitude que les MMEA correspondants dans l'échantillon anglais, alors que près de $24 \%$ des MMEA expriment un degré de certitude plus élevé que le texte source. Les traductions de Moulinié (FR1873) et de Barbier (FR1876) affichent un profil similaire. Les MMEA exprimant le même degré de certitude que les MMEA anglais correspondants représentent 67,5\% des MMEA repérés dans la traduction de Moulinié et 69,5\% de ceux repérés dans la traduction de Barbier. La traduction de Moulinié comporte également une plus grande proportion de MMEA exprimant un degré de certitude plus élevé que les MMEA anglais correspondants que la traduction de Barbier (16\% contre $12 \%)$.

\section{Discussion}

Notre étude se veut exploratoire dans la mesure où, pour des raisons de faisabilité, les 55 phrases analysées dans les quatre traductions françaises à l'étude forment un corpus restreint par rapport à l'ensemble de l'OS. Nous avons déterminé que notre échantillon présentait des différences quant au nombre et à la répartition des différents types de MMEA en comparaison au chapitre IV. Cependant, les résultats tirés de cet échantillon peuvent néanmoins nous éclairer quant aux tendances de chacun des traducteurs, car les effectifs observés autorisent l'application du test statistique $\mathrm{du} \mathrm{X}^{2}$, la plupart du temps. Lorsque la granularité des paramètres observés augmente, on constate cependant que le $\mathrm{X}^{2}$ devient de moins en moins applicable. Dans ce cas, 
notre étude nous donne des éléments concrets pour élargir le corpus et envisager des stratégies complémentaires pour : 1) confirmer les tendances observées dans d'autres parties de l'œuvre étudiée et 2) corréler plusieurs phénomènes. D’ores et déjà, les résultats que nous présentons - notamment une différence plus ou moins marquée avec le texte source et un traitement non identique des MMEA par les quatre traducteurs - apportent des premiers éléments de réponse dans l'étude des traductions françaises de l'OS.

Certains résultats concernant la comparaison des répartitions des MMEA entre chacune des traductions et l'échantillon anglais étaient attendus. En effet, ils montrent que la traduction de Royer diffère significativement du texte de départ sur le plan de la répartition des MMEA (section 6.2). Elle est également celle qui affiche la proportion la plus élevée de MMEA accentuant le degré de certitude du texte $(23,4 \%)$, en plus de comporter le deuxième plus haut total d'omissions (52). Par comparaison, Barbier réalise presque le même nombre d'omissions (53), mais fait 10 ajouts de plus (31, contre 21 pour Royer). Étonnamment, Barbier manipule le texte source autant, sinon plus, que Royer. Il faut souligner que les omissions, en plus d'affecter la répartition des MMEA entre les différents types, jouent sur le degré de certitude exprimé par la traduction. Hyland (1999: 79) affirme en effet que: «[the removal of hedges] is a major linguistic means of conferring greater certainty on propositions.» Tous ces résultats convergent vers une augmentation du degré de certitude de la traduction de Royer par rapport à l'échantillon anglais. Ceci est en accord avec les conclusions de Brisset, pour qui la traduction de Royer affiche bel et bien un degré de certitude plus élevé que son original: «[...] elle fait converger l'argumentaire darwinien vers la nécessité; c'est-à-dire qu'elle transforme l'affirmation de l'évolutionnisme en prétention absolue» (Brisset 2002: 186). Pourtant, comme le montre le tableau 10, les stratégies de traduction employées par Royer ne sont pas significativement différentes de celles de Moulinié et de Barbier. Les divergences chez Royer ont cependant davantage marqué l'imaginaire, peut-être en raison de son paratexte explosif, de la forte personnalité de la traductrice et de son état de femme.

Les résultats relatifs à la traduction de Barbier étaient, pour leur part, inattendus. En effet, celle-ci diffère, elle aussi, significativement du patron de répartition des catégories de MMEA de l'original anglais. Cette différence est même plus marquée que dans le cas de Royer (valeur du $\mathrm{X}^{2}$ plus élevée chez Barbier que chez Royer). Le degré de modalité épistémique y a aussi été modifié dans environ $30 \%$ des cas (accentuation de la certitude, 18,8\%; atténuation de la certitude, 11,7\%). Cependant, cette traduction est également celle qui comporte à la fois le plus de MMEA ajoutés (31) et le plus de MMEA omis (53). On remarque donc une modification de la modalité épistémique dans la traduction de Barbier, qui provoque une accentuation du degré de certitude de la traduction par rapport à l'anglais, bien que celle-ci soit moins importante que dans la traduction de Royer. Il est intéressant de rappeler que le texte de Barbier - seule traduction française de la $6^{\mathrm{e}}$ et dernière édition définitive de l'OS parue à l'époque darwinienne - était considéré jusqu'à récemment comme canonique dans l'histoire des traductions françaises de l'OS. On connaît peu de chose d'Edmond Barbier, qui n'a laissé qu'une préface très courte dans sa traduction et peu de notes. Tort (2005: 206) le décrit comme un angliciste et fait surtout état de ses diverses traductions, autant des ouvrages de Darwin que de ceux d'autres savants de l'époque. Nos résultats suggèrent que la traduction de Barbier s'écarte plus de l'original anglais 
qu'on aurait pu le croire, un état de fait qui a pu être camouflé par la controverse qu'a toujours suscitée la traduction de Royer.

Quant à la traduction de Moulinié, elle n'est pas significativement différente du texte source sur le plan de la répartition des MMEA. Le degré de modalité épistémique y a été modifié dans 32,6\% des cas (accentuation de la certitude, 16,3\%; atténuation de la certitude, 16,3\%). Étant donné l'équipartition entre accentuation et atténuation de la certitude, on pourrait dire qu'il n'y a aucun effet sur le degré de certitude global de la traduction. Cependant, le texte de Moulinié comporte un nombre non négligeable d'omissions, deux fois plus élevé que le nombre d'ajouts. Il en résulte une accentuation du degré de certitude dans la traduction de Moulinié, bien que moins marquée que dans le cas de Royer et de Barbier. Fait intéressant, la qualité du français écrit de Moulinié, d’origine suisse, avait été remise en question. En effet, Moulinié a été révisé et corrigé par Edmond Barbier, autant pour sa traduction de l'OS (Prum 2014: 396) que celle de The Descent of Man (1871; voir note 1). L'éditeur français Charles-Ferdinand Reinwald attribue d'ailleurs la faiblesse des ventes de cette dernière traduction en France à une piètre qualité linguistique (lettre de C.-F. Reinwald à C. Darwin datée du 14 mai 1873 $3^{39}$ ). Dans une lettre à Darwin datée du 23 avril 1867, le scientifique allemand Carl Vogt rapporte que Jean-Jacques Moulinié lui a révélé que sa mère est anglaise et qu'il «parle l'anglais comme [sa] langue maternelle ${ }^{40}$. Les raisons objectives des critiques restent à déterminer, mais on peut envisager la possibilité d'interférences linguistiques ou l'emploi de régionalismes suisses trahissant son éloignement de Paris, ou encore, une sévérité de jugement motivée par d'autres raisons. Néanmoins, en ce qui concerne le traitement des MMEA, il semble que Moulinié respecte davantage que Barbier le patron établi par le texte anglais.

Pour leur part, les résultats tirés de la traduction de Berra sont conformes aux attentes, étant donné son projet de traduction. Celui-ci est cohérent avec l'orientation adoptée pour le vaste projet de retraduction des œuvres de Darwin mené par Patrick Tort, dans lequel s'inscrit la traduction de Berra. En effet, dans le paratexte des retraductions faisant partie de ce projet, Tort critique souvent les traductions françaises contemporaines à Darwin, les qualifiant même d'«inacceptables» (Tort et Prum 2008: 164), sans cependant préciser pourquoi. Il préconise plutôt une méthode de traduction très sourcière, voire littérale par endroits. Engel-Gautier (2010), dans son étude de la retraduction de The Descent of Man par l'équipe de Tort, mentionne que ces derniers vont même jusqu'à faire violence à la langue française. Comme l'indique le tableau 7, la répartition des MMEA n'était pas significativement différente entre la traduction de Berra et l'échantillon anglais. De plus, près de $93 \%$ des MMEA repérés dans cette traduction expriment un degré de certitude équivalent aux MMEA présents en anglais. C'est cette traduction qui comportait également le moins d'omissions et le moins d'ajouts. Le texte de Berra est donc celui qui se rapproche le plus de l'original en ce qui concerne le degré de certitude. Il faut également noter que, comme le montrent le tableau 10 et la figure 3, la traduction de Berra est significativement différente des trois autres traductions pour ce qui est de la répartition des MMEA traduits, omis et ajoutés, alors que l'on n'observe pas de différence significative entre les trois traductions restantes. On remarque donc une distinction claire entre les traductions contemporaines de Darwin et la traduction moderne que comporte notre étude.

La réalisation de ce projet a également montré la nécessité de certaines rectifications méthodologiques pour des travaux futurs. Tout d'abord, l'annotation des textes 
source et cible devrait se faire en parallèle, car, souvent, la traduction elle-même met en évidence l'importance de certaines expressions linguistiques dans le texte source qui passent inaperçues si le chercheur annote ce dernier indépendamment (voir Gendron-Pontbriand, Bodain, et Vandaele 2016 pour une comparaison des annotations séquentielle et parallèle). Puis, la mise en parallèle de l'original darwinien avec ses traductions a également mis au jour la saillance d'autres phénomènes de modalité, entre autres: la modalité épistémique exprimant la conviction (les boosters [Hyland 2005], phénomène contraire aux MMEA), le rôle des temps de verbe et du mode verbal, les marqueurs d'articulation logique et l'organisation thème/rhème. En effet, nous avons observé des différences marquées entre l'original anglais et ses quatre traductions sur le plan de ces autres phénomènes discursifs. Finalement, nous mentionnons également la question du nombre et de la longueur des phrases. Nous sommes en droit de nous demander si le style de Darwin - longues phrases, multiples incises, un effet de lenteur et de prudence - ne constitue pas en lui-même un élément de MEA discursive, qui est ainsi perturbé par la fragmentation des phrases dans une traduction comme celle de Royer, pour donner un style plus rapide, plus direct.

Crisafully (2002: 27) souligne que l'opposition entre les approches empiriquesdescriptives et critiques-interprétatives constitue un obstacle à l'avancement de la traductologie. Selon lui, une éventuelle réconciliation passe par la reconnaissance de la valeur des analyses aussi bien qualitatives que quantitatives. La présente étude se base plutôt sur des méthodes quantitatives, qui, comme le signale Tymoczko (2007: 157), constituent la classe d'outils de validation la plus puissante en recherche. L'utilité des analyses quantitatives est en effet considérable, car ces dernières permettent de dégager des tendances ou des régularités dans les traductions à l'étude (Crisafully 2002: 37). Même les études littéraires, qui s'inscrivent plutôt dans une tradition critique-interprétative, accordent de plus en plus d'importance aux analyses quantitatives: «[...] the bare claim that Woolf uses a great deal of personification is without value and nearly meaningless unless it is quantified. [...] most stylistic and interpretive observations rest upon patterns, and, therefore, upon repetition» (Hoover 2013: 518-519). Notre étude a donc fourni des éléments de réponse à d'importantes questions méthodologiques et historiques. Elle révèle entre autres l'intérêt d'une approche quantitative sur corpus appliquée à des textes historiques. Elle remet également en question des idées reçues par rapport à la place de la traduction de Royer dans la suite des traductions françaises de l'OS. Nous montrons en effet que celle-ci a plus en commun avec les traductions de Moulinié et de Barbier que l'on aurait pu croire. La présente étude pourra donc servir de tremplin à d'autres explorations des différentes traductions françaises de l'OS.

\section{REMERCIEMENTS}

La présente recherche a été rendue possible grâce à la bourse de doctorat Joseph-Armand Bombardier attribuée par le Conseil de recherche en sciences humaines du Canada à Eve-Marie Gendron-Pontbriand. Nous remercions Sylvie Vandaele pour les discussions au cours du projet, Yan Bodain (Université de Sherbrooke) pour son appui en matière de programmation XML et Nathan Ménard (Université de Montréal) pour ses conseils en matière de tests statistiques. Nous remercions également Aurélien Berra (Université Paris-Ouest Nanterre La Défense), qui a gracieusement mis à notre disposition sa traduction de l'OS en format électronique. 


\section{NOTES}

1. DARWIn, Charles (1871): The descent of man, and selection in relation to sex. Londres: John Murray.

2. DARWIN, Charles (1859): On the origin of species by means of natural selection, or the preservation of favoured races in the struggle for life. $1^{\text {re }}$ éd. Londres: John Murray.

3. DARwin, Charles (1861/1862): De l'origine des espèces ou des lois du progrès chez les êtres organisés.

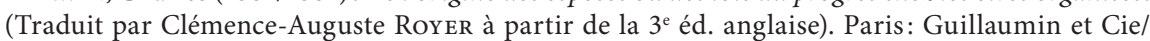
Victor Masson et Fils. DARwin, Charles (1861): On the origin of species by means of natural selection, or the preservation of favoured races in the struggle for life. $3^{\mathrm{e}}$ éd. Londres: John Murray.

4. Theodosius Dobzhansky (1900-1975) est un célèbre généticien ukrainien (émigré aux États-Unis en 1928), reconnu comme étant un des artisans de la théorie synthétique de l'évolution, élaborée dans les années 1930 et 1940 (Dreuil 2005: 1239-1255).

5. DobZhansky, Theodosius (1973): Nothing in Biology Makes Sense Except in the Light of Evolution. American Biology Teacher. 35:125-129.

6. Il est ici question des six éditions anglaises de l'OS révisées par Darwin et parues de son vivant, et non des diverses réimpressions publiées après sa mort.

7. L'Index Translationum, mis en ligne par l'UNESCO, répertorie en tout 31 langues cibles: allemand, basque, catalan, chinois, coréen, croate, danois, espagnol, français, galicien, grec moderne, hébreu, hongrois, italien, japonais, kazakh, macédonien, malayalam, mongol, néerlandais, norvégien, polonais, portugais, russe, serbe, suédois, slovaque, tchèque, tibétain et turc. Toutefois, cette liste n'est pas nécessairement complète, car la version électronique ne contient que l'information bibliographique des livres traduits et publiés entre 1979 et 2009. Consulté le 12 novembre 2013, <http:// www.unesco.org/culture/xtrans/>.

8. DARWIN, Charles (1859/1860): Het ontstaan der soorten van dieren en planten door middel van natuurkeus ofhet bewaard blijven van bevoorregte rassen in den strijd des levens. (Traduit par Tiberius Comelius WinkLer à partir de la $1^{\text {re }}$ éd. anglaise, 1859). Haarlem: Kruseman.

9. DARWIN, Charles (1859/2000): Over het ontstaan van soorten door middel van natuurlijke selectie, ofHet behoud van bevoordeelde rassen in de strijd om het leven. (Traduit par Ludo Hellemans à partir de la $1^{\text {re }}$ éd. anglaise, 1859). Amsterdam: Nieuwezijds.

10. DARwin, Charles (1869/1873): L'origine des espèces au moyen de la sélection naturelle, ou La lutte pour l'existence dans la nature. (Traduit par Jean-Jacques Moulinié à partir de la $5^{\mathrm{e}}$ éd. anglaise). Paris: C. Reinwald et Cie. DARwin, Charles (1869): On the origin of species by means of natural selection, or the preservation of favoured races in the struggle for life. $5^{\mathrm{e}}$ éd. Londres: John Murray.

11. DARWIn, Charles (1872/1876): L'origine des espèces au moyen de la sélection naturelle ou La lutte pour l'existence dans la nature. (Traduit par Edmond BARBIER à partir de la $6^{\mathrm{e}}$ éd. [6a] anglaise). Paris: C. Reinwald et Cie. DARwin, Charles (1872): The origin of species by means of natural selection, or the preservation of favoured races in the struggle for life. $6^{\mathrm{e}}$ éd. [6a]. Londres: John Murray.

12. DARwin, Charles (1876/2009): L'origine des espèces - Par le moyen de la sélection naturelle, ou la préservation des races favorisées dans la lutte pour la vie. (Traduit par Aurélien BERRA à partir de la $6^{\mathrm{e}}$ éd. [6b] anglaise). In: Patrick TorT et Michel Prum, dir. Euvres complètes - Charles Darwin. Paris: Honoré Champion. DARwIn, Charles (1876): The origin of species by means of natural selection, or the preservation of favoured races in the struggle for life. $6^{\mathrm{e}}$ éd. [6b]. Londres: John Murray.

13. Ce que Portner appelle «phrase» (sentence) correspond en fait à la proposition (clause).

14. Portner (2009: 3) souligne que les modalités sous-phrastique et phrastique se chevauchent fréquemment, et donc qu'il est difficile de les distinguer de façon définitive.

15. Phénomène pragmatique où une phrase manifestant de la modalité phrastique affecte l'interprétation des phrases modalisées subséquentes (Portner 2009: 8).

16. Exemples adaptés de Portner (2009: 2-3).

17. Tableau établi à partir de Hyland (1999: 102-147)

18. Tableau repris de Gendron-Pontbriand (2013:36).

19. DARWIN, Charles (1860): On the origin of species by means of natural selection, or the preservation of favoured races in the struggle for life. $2^{\mathrm{e}}$ éd. Londres: John Murray.

20. DARWIN, Charles (1866): On the origin of species by means of natural selection, or the preservation of favoured races in the struggle for life. $4^{\mathrm{e}}$ éd. Londres: John Murray.

21. Les éditions $6 \mathrm{a}$ et $6 \mathrm{~b}$ ne diffèrent que par quelques corrections typographiques et orthographiques mineures, apportées par Darwin lui-même. Le texte n'ayant pas été modifié, l'édition de 1876 n'est pas envisagée comme distincte de celle de 1872.

22. Tableau adapté de Gendron-Pontbriand (2013: 37). 
23. DARwin, Charles (1859/1992/2008): L'Origine des espèces au moyen de la sélection naturelle ou la préservation des races favorisées dans la lutte pour la vie. (Traduction de la $1^{\text {re }}$ éd. anglaise, 1859, établie par Daniel BeCQUemont à partir de la traduction de la $6^{\mathrm{e}}$ éd., 1872, anglaise par Edmond Barbier) Daniel Becquemont et Jean-Marc Drouin, dir. Paris: Flammarion.

24. Darwin, Charles (1859/2013): L'Origine des espèces. (Traduit par Thierry Hoquet à partir de la $1^{\text {re }}$ éd. anglaise, 1859). Paris: Seuil.

25. DARwin, Charles (1861/1866): De l'origine des espèces par sélection naturelle ou des lois de transformation des êtres organisés. (Traduit par Clémence-Auguste Royer à partir de la $3^{e}$ éd. anglaise, 1861). $2^{\mathrm{e}}$ éd. Paris: Guillaumin et Cie/Victor Masson et Fils.

26. DARWIn, Charles (1861/1870): De l'origine des espèces par sélection naturelle ou des lois de transformation des êtres organisés. (Traduit par Clémence-Auguste RoYer à partir de la $3^{\mathrm{e}}$ éd. anglaise, 1861). $3^{\mathrm{e}}$ éd. Paris: Guillaumin et Cie/Victor Masson et Fils.

27. DARwin, Charles (1861/1883 ?): De l'origine des espèces par sélection naturelle ou des lois de transformation des êtres organisés. (Traduit par Clémence-Auguste Royer à partir de la $3^{\mathrm{e}}$ éd. anglaise, 1861). 4 éd. Paris: Flammarion.

28. DARWIn, Charles (1861/s.d.): De l'origine des espèces par sélection naturelle ou des lois de transformation des êtres organisés. (Traduit par Clémence-Auguste Royer à partir de la $3^{\mathrm{e}}$ éd. anglaise, 1861). $5^{\text {e }}$ éd. Paris: Marpon et Flammarion.

29. Les quatrième et cinquième éditions de la traduction de Royer n'ont pu être incluses dans cette comparaison, car elles ne sont disponibles qu'en format papier à la Bibliothèque du Musée de l'Homme (Paris, France).

30. Hoquet (Darwin 1859/2013: 10) qualifie en effet la traduction de Becquemont d' "étrange édifice».

31. van Wyhe, John, dir. (2002- ...): The Complete Works of Charles Darwin Online. Consulté le 15 juillet 2016, <http://darwin-online.org.uk/>.

32. Reproduit de Gendron-Pontbriand (2013).

33. La méthode utilisée a été établie dans le groupe de recherche BiomeTTico pour le repérage de métaphores conceptuelles dans des corpus comparables (Vandaele et Lubin 2005; Vandaele et Boudreau 2006; Lubin 2006; Labelle 2009).

34. Le $\mathrm{X}^{2}$ est un test non paramétrique qui permet de mesurer l'écart entre une distribution observée et une distribution dite théorique. Pour ce faire, le test permet l'examen de deux hypothèses. D'une part, l'hypothèse nulle $\left(\mathrm{H}_{0}\right)$ prédit que la différence entre les distributions est nulle ou non significative. D'autre part, l'hypothèse alternative $\left(\mathrm{H}_{1}\right)$ affirme qu'il existe une différence significative entre les deux distributions. Comme l'explique Chanquoy (2005:24), «[...] la décision de rejeter ou de conserver l'hypothèse nulle dépend de la valeur du $\mathrm{X}^{2}$ calculée comparée à celle du $\mathrm{X}^{2}$ critique lue dans la table, en fonction du seuil fixé et des degrés de liberté [...].» Si le $\mathrm{X}^{2}$ calculé est inférieur au $\mathrm{X}^{2}$ critique, on ne peut pas rejeter $\mathrm{H}_{0}$, et vice versa. La valeur du $\mathrm{X}^{2}$ critique correspond à une probabilité, appelée «seuil de confiance» (ou $\alpha$ ), qui représente le risque de rejeter $\mathrm{H}_{0}$ à tort. Le seuil généralement admis est de $5 \%$, ou $\alpha=0,05$ (Chanquoy $2007: 247$ ). À noter que la probabilité $p$ de rejeter $\mathrm{H}_{0}$ à tort peut être déterminée pour toute valeur de $\mathrm{X}^{2}$ calculée. Ainsi, la valeur du $\mathrm{X}^{2}$ et la valeur $p$ correspondante permettent de chiffrer l'écart entre deux distributions: plus la valeur du $\mathrm{X}^{2}$ est élevée et la valeur $p$ est basse, plus la différence entre les distributions est marquée (et inversement).

35. Akritas, Michael (Dernière mise à jour: 5 novembre 2006): Chi-Square Distribution Table. Consulté le 10 mai 2016, <http://sites.stat.psu.edu/ mga/401/tables/Chi-square-table.pdf>.

36. Dinov, Ivo (Dernière mise à jour : 5 mars 2015): High-Precision Distribution Calculators. Consulté le 20 juin 2016, <http://www.socr.ucla.edu/Applets.dir/Normal_T_Chi2_F_Tables.htm>.

37. Ce regroupement a été motivé par le fait que ces deux types de MMEA sont l'un et l'autre des marqueurs de MEA syntaxique.

38. Ce graphique a été réalisé à l'aide du logiciel Distance de Brian Kinghorn (http://bkinghor.une. edu.au/distance.htm).

39. Cité dans Vandaele et Gendron-Pontbriand (2015: 269).

40. Lettre de C.-F. Reinwald à C. Darwin, le 23 avril 1867. Consultée le $1^{\text {er }}$ avril 2016, <http://www. darwinproject.ac.uk/entry-5512>. 


\section{RÉFÉRENCES}

Brisset, Annie (2002): Clémence Royer, ou Darwin en colère. In: Jean DelisLe, dir. Portraits de traductrices. Ottawa: Presses de l'Université d'Ottawa, 173-203.

ChANQuoy, Lucile (2005): Statistiques appliquées à la psychologie et aux sciences humaines et sociales. Paris: Hachette.

Conry, Yvette (1974): L'introduction du darwinisme en France au XIXe siècle. Paris: Vrin.

Crisafully, Edoardo (2002): The Quest for an Eclectic Methodology of Translation Description. In: Theo Hermans, dir. Crosscultural Transgressions. Research Models in Translation Studies II. Historical and Ideological Issues. Manchester: St. Jerome, 26-43.

Crismore, Avon et Farnsworth, Rodney (1989): Mr. Darwin and His Readers: Exploring Interpersonal Metadiscourse as a Dimension of Ethos. Rhetoric Review. 8(1):91-112.

DARWIn, Francis (1887): The life and letters of Charles Darwin, including an autobiographical chapter. Vol. 3. Londres: John Murray.

DAY, Claudine (2008): Modalité et modalisation dans la langue. Paris: L’Harmattan.

Desclés, Jean-Pierre (2003) : Interactions entre les valeurs de pouvoir, vouloir, devoir. In: Merete Birkelund, Gerhard Boysen et Poul Søren KjÆrsgaard, dir. Aspects de la modalité. Actes du colloque international sur la modalité. Tübingen: Max Niemeyer Verlag, 49-66.

Dreuil, Daniel (1996): Néo-darwinisme. In: Patrick TorT, dir. Dictionnaire du darwinisme et de l'évolution. Paris: Presses Universitaires de France, 3165-3184.

Engel-Gautier, Carolyn (2010): Retranslating scientific works: The case of Darwin's The Descent of Man. In: David Banks, dir. Aspects diachroniques du texte de spécialité. Paris: L'Harmatan, 35-52.

Gendron-Pontbriand, Eve-Marie (2013): Modes de conceptualisation métaphoriques et théorie de l'évolution: analyse textuelle et traduction. Mémoire de maitrise non publié. Montréal: Université de Montréal.

Gendron-Pontbriand, Eve-Marie, Bodain, Yan et Vandaele, Sylvie (2016): Deux méthodes d'annotation de la conceptualisation métaphorique dans un corpus parallèle: On the Origin of Species de Charles Darwin. In: Damon Mayaffre, Céline Poudat, Laurent Vanni, et al., dir. JADT 2016: Actes des $13^{\text {es }}$ Journées internationales d'Analyse statistique des Données Textuelles. (13 ${ }^{\text {es }}$ Journées internationales d'Analyse statistique des Données Textuelles, Nice, France, 7-10 juin 2016). Vol. II. Nice: Centre national de la recherche scientifique/Université Nice Sophia Antipolis, 719-730.

Hoover, David L. (2013): Quantitative Analysis and Literary Studies. In: Susan Schreibman et Ray Siemens, dir. A Companion to Digital Literary Studies. Oxford: Blackwell, 517-533.

Hoquet, Thierry (2013): The Evolution of the Origin (1859-1872). In: Michael Ruse, dir. The Cambridge Encyclopedia of Darwin and Evolutionary Thought. Cambridge: Cambridge University Press, 158-164.

Hyland, Ken (1999): Hedging in Scientific Articles. Amsterdam/Philadelphie: John Benjamins.

Hyland, Ken (2005): Metadiscourse. Exploring Interaction in Writing. Londres/New York: Continuum.

LABelle, Mélanie (2009): Les réseaux lexicaux nominaux témoignant de la conceptualisation métaphorique en anatomie. Mémoire de maîtrise non publié. Montréal: Université de Montréal.

Larreya, Paul (2004): L'expression de la modalité en français et en anglais (domaine verbal). Revue belge de philologie et d'histoire. 82(3):733-762.

Lubin, Leslie (2006): Études des métaphores conceptuelles utilisées dans la description des structures anatomiques. Mémoire de maîtrise non publié. Montréal: Université de Montréal.

Miles, Sara Joan (1988): Evolution and Natural Law in the Synthetic Science of Clémence Royer. Thèse de doctorat non publiée. Chicago: University of Chicago.

Miles, Sara Joan (1989): Clémence Royer et De l'Origine des espèces: traductrice ou traitresse. Revue de synthèse. 4:61-83.

MülLER, Charles (1968): Initiation à la statistique linguistique. Paris: Larousse. 
Olohan, Maeve et Salama-Carr, Myriam (2011): Translating Science. The Translator. 17(2):179-188.

Portner, Paul (2009): Modality. Oxford: Oxford University Press.

Prum, Michel (2014): Charles Darwin's First French Translations. In: Thoms F. GLICK et Elinor ShAfFer. The Literary and Cultural Reception of Charles Darwin in Europe. Vol. 4. Londres/ New York: Bloomsbury, 391-399.

RUSE, Michael (2000): Metaphor in evolutionary biology. Revue internationale de philosophie. 54(214):593-619.

Tort, Patrick (2005) : Dictionnaire du darwinisme et de l'évolution. Paris: Presses Universitaires de France.

TorT, Patrick et Prum, Michel (2008): Avertissement sur la traduction. In: Charles DARwin. La variation des animaux et des plantes à l'état domestique. (Traduit par Aurélien BERRA) Euvres complètes - Charles Darwin. Patrick TorT et Michel PrUM, dir. Genève: Slatkine, 164.

Tүмосzко, Maria (2007): Enlarging Translation, Empowering Translators. Manchester: St. Jerome. VANDAELE, Sylvie (2009): Les modes de conceptualisation du vivant: une approche linguistique. In: Sylvain David, Janusz Prychodzen et François-Emmanuël Boucher, dir. Que peut la métaphore? Histoire, savoir et poétique. Paris: L'Harmattan, 187-207.

VAndaele, Sylvie et Boudreau, Sylvie (2006): Annotation XML et interrogation de corpus pour l'étude de la conceptualisation métaphorique. In: Jean-Marie Viprey, dir. JADT'06: Actes des $8^{e s}$ Journées internationales d'Analyse statistique des Données Textuelles. ( $8^{\text {es }}$ Journées internationales d'Analyse statistique des Données Textuelles, Université de Franche-Comté, Besançon, 19-21 avril 2006). Vol. 2. Besançon: Presses universitaires de Franche-Comté, 951-959.

Vandaele, Sylvie et Gendron-Pontbriand, Eve-Marie (2015): Des "vilaines infidèles» à la postérité: traduction et retraduction de l'œuvre de Charles Darwin. In: Julia Pinilla et Brigitte LépINeTte, dir. Traducción y diffusión de ciencia y de la técnica en España en los siglos XVIII y XIX. València: Universitat de València, 249-275.

Vandaele, Sylvie et Lubin, Leslie (2005): Approche cognitive de la traduction des langues de spécialité: vers une systématisation de la description de la conceptualisation métaphorique. Meta. 50(2):415-431.

Vandepitte, Sonia, Vandenbussche, Liselotte et Algoet, Brecht (2011): Travelling Certainties: Darwin's Doubts and Their Dutch Translations. The Translator. 17(2):275-299.

Vihla, Minna (1999): Medical Writing: Modality in Focus. Atlanta/Amsterdam: Rodopi.

von Fintel, Kai et Gillies, Anthony (2007) : An Opinionated Guide to Epistemic Modality. In: Tamar Szabo Gendeer et John Hawthorne, dir. Oxford Studies in Epistemology. Vol. 2. Oxford: Oxford University Press, 32-63.

Young, Robert Maxwell (1985): Darwin's Metaphor: Nature's Place in Victorian Culture. Cambridge: Cambridge University Press.

Ziegeler, Debra (2006) : Mood and Modality in Grammar. In: Keith Brown, dir. Encyclopedia of Language \& Linguistics. $2^{\mathrm{e}}$ éd. Oxford: Elsevier, 259-267. 


\section{ANNEXE}

Le tableau suivant fournit des exemples tirés de notre corpus, montrant les MMEA dans le texte anglais de 1861 et leurs équivalents dans les quatre traductions françaises étudiées. Le symbole ${ }^{\circ} \mathrm{ME}$ (degré de modalité épistémique) fait référence au degré de certitude exprimé par le MMEA français par rapport au MMEA correspondant dans l'original anglais.

\begin{tabular}{|c|c|c|c|}
\hline${ }^{\circ} \mathrm{ME}$ & Texte anglais & Traduction & Texte français \\
\hline Plus & $\begin{array}{l}\text { "[...] I can see no greater difficulty } \\
\text { in this being effected through } \\
\text { natural selection }[\ldots] »(\text { p. 91) }\end{array}$ & \multirow{3}{*}{ FR1862 } & $\begin{array}{l}\text { «[...] il est aussi aisé à l'élection naturelle } \\
\text { de produire un semblable } \\
\text { perfectionnement [...]» (p. 123) }\end{array}$ \\
\hline Égal & $\begin{array}{l}\text { «[..] so by generation I believe it } \\
\text { has been with the great Tree of Life } \\
{[\ldots] »(\mathrm{p} .147)}\end{array}$ & & $\begin{array}{l}\text { "[...] ainsi, par une suite de générations } \\
\text { non interrompues, il en a été, je crois, du } \\
\text { grand arbre de la vie }[\ldots] »(\text { p. } 190)\end{array}$ \\
\hline Moins & $\begin{array}{l}\text { "One large group will slowly } \\
\text { conquer another large group [...]» } \\
\text { (p. 131) }\end{array}$ & & $\begin{array}{l}\text { «Un groupe déjà puissant pourra seul } \\
\text { vaincre un autre groupe }[\ldots] »(\text { p. } 171)\end{array}$ \\
\hline Plus & $\begin{array}{l}\text { "[...] and the largeness of any group } \\
\text { shows that its species have inherited } \\
\text { from a common ancestor some } \\
\text { advantage in common.» (p. 131) }\end{array}$ & \multirow{3}{*}{ FR1873 } & $\begin{array}{l}{[\ldots] \text { et l'extension qu'a prise un groupe }} \\
\text { donné prouve que les espèces qui le } \\
\text { constituent ont hérité toutes ensemble } \\
\text { d'un commun ancêtre de quelque } \\
\text { condition favorable.» (p. 128) }\end{array}$ \\
\hline Égal & $\begin{array}{l}\text { "I conclude that for terrestrial } \\
\text { productions of a large continental } \\
\text { area }[\ldots] »(\text { p. 113) }\end{array}$ & & $\begin{array}{l}\text { «[...] je conclus que c'est dans une vaste } \\
\text { étendue de continent }[. . .] »(\text { p. 112) }\end{array}$ \\
\hline Moins & $\begin{array}{l}\text { "[...] and we may at least safely } \\
\text { infer that diversification of } \\
\text { structure, amounting to new } \\
\text { generic differences, would be } \\
\text { profitable to them.» (p. 121) }\end{array}$ & & $\begin{array}{l}\text { "[...] et nous sommes fondés à admettre, } \\
\text { que des diversifications dans leur } \\
\text { structure, ayant une valeur générique, } \\
\text { auraient pu leur être avantageuses.» } \\
\text { (p. 120) }\end{array}$ \\
\hline Plus & $\begin{array}{l}\text { "[...] in such cases there might be } \\
\text { retrogression in the scale of } \\
\text { organisation.» (p. 134) }\end{array}$ & \multirow{3}{*}{ FR1876 } & $\begin{array}{l}\text { «[..] dans ce cas, il y aurait une } \\
\text { rétrogradation réelle dans l'échelle de } \\
\text { l'organisation.» (p. 134) }\end{array}$ \\
\hline Égal & $\begin{array}{l}\text { "From these several considerations } \\
\text { I think it inevitably follows [...]» } \\
\text { (p. 116) }\end{array}$ & & $\begin{array}{l}\text { «Je crois que ces différentes } \\
\text { considérations nous conduisent à la } \\
\text { conclusion inévitable }[\ldots] »(\text { p. 118) }\end{array}$ \\
\hline Moins & $\begin{array}{l}\text { "Natural Selection will modify the } \\
\text { structure of the young in relation to } \\
\text { the parent, and of the parent in } \\
\text { relation to the young.» (p. 91) }\end{array}$ & & $\begin{array}{l}\text { «La sélection naturelle peut modifier la } \\
\text { conformation du jeune relativement aux } \\
\text { parents et celle des parents relativement } \\
\text { aux jeunes.» (p. 92) }\end{array}$ \\
\hline Plus & $\begin{array}{l}\text { "[...] I can see no limit to the } \\
\text { amount of change [...] which may } \\
\text { be effected in the long course of } \\
\text { time by nature's power of } \\
\text { selection.» (p. 115) }\end{array}$ & \multirow{3}{*}{ FR2009 } & $\begin{array}{l}\text { «[...] je ne vois aucune limite à }[\ldots] \text { la } \\
\text { complexité des coadaptations [...] qui ont } \\
\text { pu être obtenus dans la longue suite des } \\
\text { temps grâce au pouvoir de sélection que } \\
\text { possède la nature [...]» (p. 393) }\end{array}$ \\
\hline Égal & $\begin{array}{l}\text { "[...] we may gain some crude idea } \\
\text { in what manner some of the natives } \\
\text { would have to be modified [...]» } \\
\text { (p. 121) }\end{array}$ & & $\begin{array}{l}\text { «[...] nous pouvons grossièrement nous } \\
\text { faire une idée de la manière dont certains } \\
\text { des habitants d'origine devraient se } \\
\text { modifier [...]» (p. 399) }\end{array}$ \\
\hline Moins & $\begin{array}{l}\text { "Natural selection acts solely } \\
\text { through the preservation of } \\
\text { variations in some way } \\
\text { advantageous, which consequently } \\
\text { endure.» (p. 115) }\end{array}$ & & $\begin{array}{l}\text { «La sélection naturelle agit uniquement } \\
\text { par le biais de la préservation de } \\
\text { variations avantageuses en quelque } \\
\text { manière, lesquelles par conséquent } \\
\text { tendent à persister.» (p. 393) } \\
\end{array}$ \\
\hline
\end{tabular}

